\title{
Glutamatergic Afferents of the Ventral Tegmental Area in the Rat
}

\author{
Stefanie Geisler, ${ }^{1}$ Christian Derst, ${ }^{2}$ Rüdiger W. Veh, ${ }^{2}$ and Daniel S. Zahm ${ }^{1}$ \\ ${ }^{1}$ Department of Pharmacological and Physiological Science, Saint Louis University School of Medicine, St. Louis, Missouri 63104, and ${ }^{2}$ Institute for \\ Integrative Neuroanatomy, Charité Universtiätsmedizin Berlin, 10098 Berlin, Germany
}

Glutamatergic inputs to the ventral tegmental area (VTA), thought crucial to the capacity of the VTA to detect and signal stimulus salience, have been reported to arise in but a few structures. However, the afferent system of the VTA comprises very abundant neurons within a large formation extending from the prefrontal cortex to the caudal brainstem. Neurons in nearly all parts of this continuum may be glutamatergic and equivalently important to VTA function. Thus, we sought to identify the full range of glutamatergic inputs to the VTA by combining retrograde transport of wheat germ agglutinin-bound gold after injections into the VTA with nonisotopic in situ hybridization of the vesicular glutamate transporters (VGLUTs) 1, 2, and 3. We found glutamatergic neurons innervating the VTA in almost all structures projecting there and that a majority of these are subcortical and VGLUT2 mRNA positive. The tremendous convergence of glutamatergic afferents from many brain areas in the VTA suggests that (1) the function of the VTA requires integration of manifold and diverse bits of information and (2) the activity of the VTA reflects the ongoing activities of various combinations of its afferents.

Key words: VTA; excitatory; VGLUT; connections; hypothalamus; reward

\section{Introduction}

The irregular firing of ventral tegmental area (VTA) neurons changes to a high-frequency burst pattern in response to rewardpredicting stimuli and novelty (Schultz, 1986, 1998; Hyland et al., 2002). The resulting increases in dopamine release in VTA target areas (1) serve as learning signals that attach biological significance to otherwise neutral environmental stimuli (Wise, 2004) and (2) enlist appropriate behavioral responses.

Glutamatergic afferents play a key role in regulating VTA cell firing. Activation of glutamatergic afferents and VTA infusions of glutamate receptor agonists increase the firing rates of dopamine neurons and induce burst firing in vivo (Grace and Bunney, 1984; Johnson et al., 1992; Chergui et al., 1993; Murase et al., 1993). Blockade of VTA ionotropic glutamate receptors potently attenuates the burst activity of VTA neurons (Charlety et al., 1991; Chergui et al., 1993). Glutamatergic actions in the VTA are critical to the effects of drugs of abuse. Establishment of conditioned place preference for morphine and cocaine (Harris and AstonJones, 2003; Harris et al., 2004) and reinstatement of cocaine seeking elicited by stimulating the subiculum (Vorel et al., 2001)

\footnotetext{
Received Jan. 3, 2007; revised April 23, 2007; accepted April 23, 2007.

This work was supported by United States Public Health Service Grants NIH-DA-15207 and NS-23805. We thank Dr. Medha Gautam for her kind help with the transformation of bacteria.

Correspondence should be addressed to either of the following: Dr. Stefanie Geisler, National Institute on Drug Abuse, Intramural Research Program, Behavioral Neuroscience Branch, Building C, 5500 Nathan Shock Drive, Baltimore, MD 21224, E-mail: GeislerS@mail.nih.gov; or Dr. Daniel S.Zahm, Department of Pharmacological and Physiological Science, Saint Louis University School of Medicine, 1411 South Grand Boulevard, St. Louis, M0 63104, E-mail:zahmds@slu.edu.

DOI:10.1523/JNEUROSCI.0012-07.2007

Copyright $\odot 2007$ Society for Neuroscience $\quad 0270-6474 / 07 / 275730-14 \$ 15.00 / 0$
}

or a priming injection of cocaine (Sun and Rebec, 2005) is blocked by VTA infusions of glutamate receptor antagonists.

Neuroanatomical (Sesack and Pickel, 1992; Charara et al., 1996; Omelchenko and Sesack, 2005) and electrophysiological (Murase et al., 1993; Georges and Aston-Jones, 2001, 2002; Floresco et al., 2003; Lodge and Grace, 2006a) evidence indicates that excitatory afferents of the VTA arise in the prefrontal cortex, bed nucleus of the stria terminalis, and laterodorsal and pedunculopontine tegmental nuclei, suggesting that VTA neuron activity is mainly controlled by few structures. However, the afferent system of the VTA comprises very abundant neurons within a nearly continuous formation extending from the prefrontal cortex to the caudal brainstem (Geisler and Zahm, 2005, 2006b). VTAprojecting neurons in nearly all parts of this continuum may be glutamatergic.

We set out to identify the full system of glutamatergic inputs of the VTA by combining retrograde transport of wheat germ agglutinin (WGA)-bound gold after injections into the VTA with nonisotopic in situ hybridization using probes specific for the vesicular glutamate transporters (VGLUTs) 1, 2, and 3. VGLUTs transport glutamate into synaptic vesicles (Fremeau et al., 2004; Takamori, 2006). The three VGLUTs share extensive sequence homology and similar affinities for glutamate (Takamori, 2006, and references therein). VGLUT1 and VGLUT2 are confined to axonal terminals and have been unambiguously established as markers of glutamatergic neurons (Bellocchio et al., 2000; Takamori et al., 2000, 2001; Fremeau et al., 2001; Herzog et al., 2001; Varoqui et al., 2002). The recently identified VGLUT3 has been observed in axonal terminals but also in somata and dendrites and in cell populations not conventionally considered to release glutamate (Fremeau et al., 2002; Gras et al., 2002; Schafer et al., 
2002). Insofar as VGLUT3 is abundantly expressed in axon terminals in the VTA (Herzog et al., 2004), consistent with a role in axonal exocytotic glutamate release there, an examination of the distribution of VGLUT3 mRNA-positive, VTA-projecting neurons was included in the present analysis.

\section{Materials and Methods}

All experiments were performed in accordance with guidelines published in the National Institutes of Health Guide to the Care and Use of Laboratory Animals.

Experimental protocols were reviewed and approved by the Animal Care Committee and monitored by the Department of Comparative Medicine of the Saint Louis University School of Medicine. The animals were maintained on a $12 \mathrm{~h}$ light/dark cycle and given food and water ad libitum. Before surgeries, they were housed in group cages and, afterward, individually. If not indicated otherwise, chemicals were purchased from Sigma (St. Louis, MO).

Tracer injections. Male Sprague Dawley rats (Harlan, Indianapolis, IN), weighing $280-350 \mathrm{~g}$, were deeply anesthetized by intraperitoneal injections of a mixture consisting of $45 \%$ ketamine ( $100 \mathrm{mg} / \mathrm{ml}), 35 \%$ xylazine $(20 \mathrm{mg} / \mathrm{ml})$, and $20 \%$ saline at a dose of $0.16 \mathrm{ml} / 100 \mathrm{~g}$ body weight and placed into a Kopf Instruments (Tujunga, CA) stereotaxic instrument. A small volume ( $200 \mathrm{nl}$ ) of the retrograde tracer WGA-apo HRP-gold (E-Y Laboratories, San Mateo, CA) was slowly injected via air pressure into the VTA and, for control experiments, into areas adjacent to it, using $1.0 \mathrm{~mm}$ glass pipettes pulled to outer tip diameters of $45-50$ $\mu \mathrm{m}$. The selection of stereotaxic coordinates was guided by the atlas of Paxinos and Watson (1998). After surgery, the rats received 0.9\% saline subcutaneously and were kept warm until fully recovered from anesthesia.

After $3 \mathrm{~d}$ of survival, the rats were again deeply anesthetized as described and perfused transaortically with $4 \%$ paraformaldehyde and $2.5 \%$ sucrose in $0.1 \mathrm{M}$ phosphate buffer $(\mathrm{PB})$, preceded by a solution containing $2.5 \%$ sucrose, $0.5 \%$ procaine, and $0.81 \% \mathrm{NaCl}$ in $0.01 \mathrm{M} \mathrm{PB}$, all at $\mathrm{pH}$ 7.4. The brains were removed, placed in fresh fixative for $4 \mathrm{~h}$, and cryoprotected in $25 \%$ sucrose overnight. Subsequently, each brain was frozen in dry ice and cut with a sliding microtome into five adjacent series of 50- $\mu \mathrm{m}$-thick sections in the coronal plane. Sections were collected from the frontal pole to the rostral medulla oblongata resulting in 54-59 sections per series. To verify that no glutamatergic VTAprojecting neurons were present caudal to the rostral medulla, sections of three brains were collected through the rostral spinal cord, which resulted in $77-85$ sections per series. All sections in a series were reacted at the same time.

Silver intensification of colloidal gold particles. Before and subsequent to in situ hybridization processing, the sections were thoroughly rinsed in $0.1 \mathrm{M}$ PB and immersed for $30 \mathrm{~min}$ in a mixture of solutions $\mathrm{A}$ and $\mathrm{B}$ from the IntensE M Silver Enhancement kit (Amersham Biosciences, Piscataway, $\mathrm{NJ}$ ), which was followed by rinses in $0.1 \mathrm{M} \mathrm{PB}$.

Probe generation. cDNAs of VGLUT1 (NM_053859; 717 bp), VGLUT2 (NM_053427; 688 bp), and VLGUT3 (NM_153725, 657 bp) were amplified by PCR from a rat brain cDNA library using the following primers: VGLUT1, 5'-GTGCGAAAGCTGATGAACTG-3' and 5'-GAGAAGGAGAGAGGGCTGGT-3'; VGLUT2, 5'-TTGGTGCAATGACGAAGAAC-3' and 5'-AGGATGACATGTGCCAACTG-3'; VGLUT3, 5'-

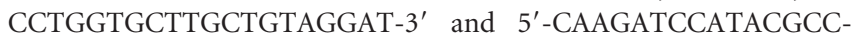
CATCT- $3^{\prime}$. The PCR products were sequenced and cloned into a pGEM-T vector (Promega, Madison, WI). The sequences encode parts of the C-terminal sequences and $3^{\prime}$ untranslated regions of the respective VGLUTs and had overall homologies below $40 \%$ to each other and no homologies to any other known rat genes. Digoxigenin-uridine triphosphate-labeled antisense and sense probes were generated by linearizing the plasmids with NotI and SacII (New England Biolabs, Ipswich, MA) and transcribing with T7 and SP6 RNA polymerases (Roche, Indianapolis, IN), respectively. The DNAs were run on $1 \%$ agarose (Invitrogen, Carlsbad, CA) gels before and after the digest, and the concentrations were measured with UV spectrophotometry (Spectronic Uni-
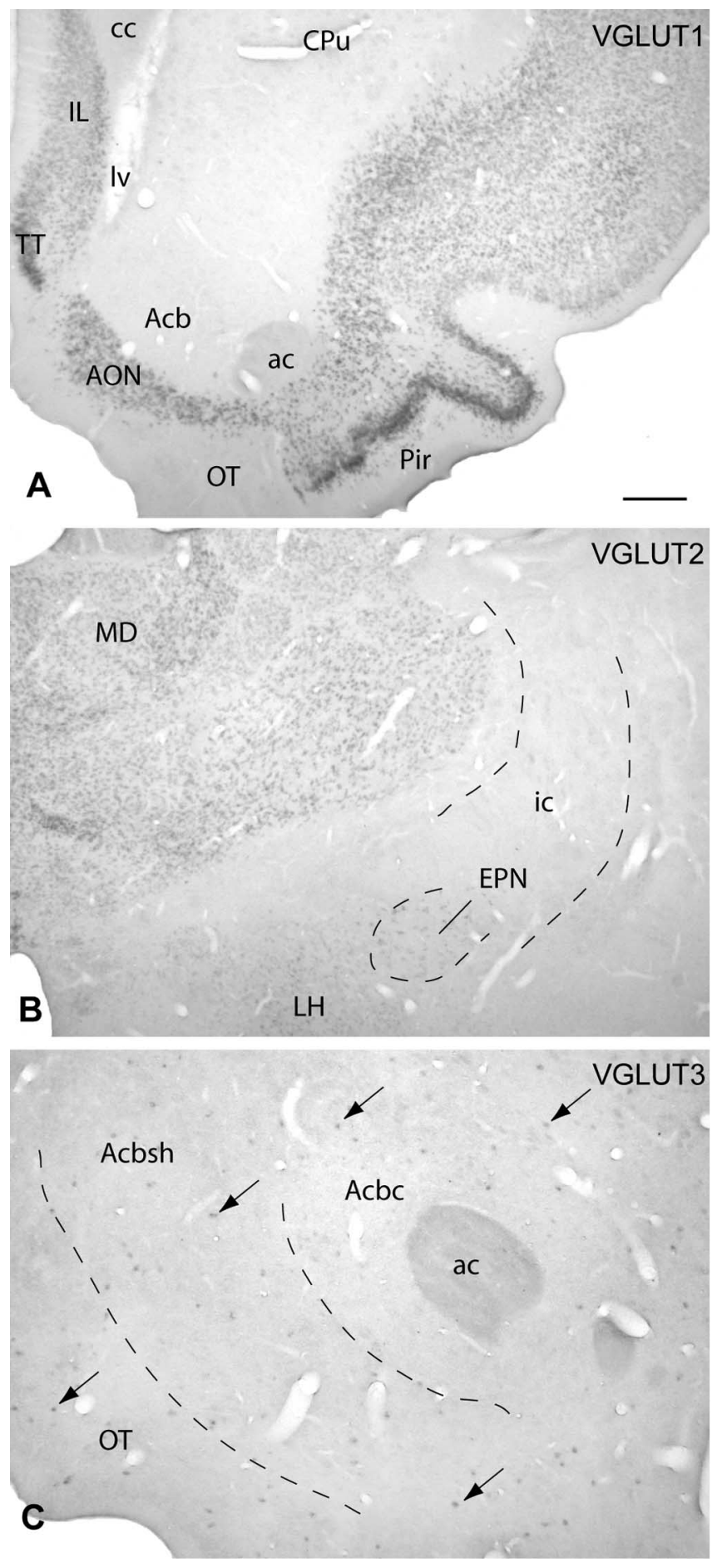

Figure 1. Micrographs illustrating the binding of probes complementary to VGLUT1, VGLUT2, and VGLUT3 mRNA mostly in distinct parts of the brain. A, VGLUT1 mRNA was predominantly expressed by neurons in cortex [e.g., in the infralimbic (IL) and piriform (Pir) areas and anterior olfactory nucleus (AON)], whereas most subcortical structures were devoid of VGLUT1 mRNA binding, as apparent in the caudate-putamen ( $\mathrm{CPu})$, accumbens ( $\mathrm{Acb})$, and olfactory tubercle (0T). TT, Taenia tecta. $\boldsymbol{B}$, In contrast, VGLUT2 mRNA was mainly expressed by neurons in subcortical structures, such as, for example, the mediodorsal thalamic nucleus (MD), lateral hypothalamic area (LH), and entopeduncular nucleus (EPN). C, VGLUT3 mRNA (arrows) was present in discrete cell populations [e.g., in the accumbens core (Acbc) and shell (Acbsh)]. ac, Anterior commissure; cc, corpus callosum; lv, lateral ventricle; ic, internal capsule. Scale bar: (in A) $A, 500 \mu \mathrm{m} ; B, 400 \mu \mathrm{m} ; C, 200 \mu \mathrm{m}$. 
cam, Cambridge, UK). The concentrations of digoxigenin-labeled RNA probes were determined by dot blot (Roche) and UV spectrophotometry.

In situ hybridization. Hybridizations were performed as described in detail recently (Geisler and Zahm, 2006b). Briefly, after immersing entire series of free-floating sections for $15 \mathrm{~min}$ in acetic anhydride ( $1.5 \%$ triethanolamine, $0.42 \% \mathrm{HCl}, 0.25 \%$ acetic anhydride in $\mathrm{H}_{2} \mathrm{O}$ ), the series were placed for $30 \mathrm{~min}$ in hybridization buffer (50\% formamide, $300 \mathrm{~mm}$ sodium chloride, 20 mм Tris, pH 7.4, 1 mм EDTA, 10\% dextran sulfate, and $1 \times$ Denhardt's reagent) and thereafter into hybridization buffer containing $0.1 \%$ SDS, $0.1 \%$ sodium thiosulfate, salmon sperm DNA $(0.1 \mathrm{mg} / \mathrm{ml})$, yeast total RNA $(0.25 \mathrm{mg} / \mathrm{ml})$, yeast tRNA $(0.25 \mathrm{mg} / \mathrm{ml})$, and the cRNA antisense and sense probes for VGLUTs 1 and 2 (at a concentration of 500 $\mathrm{ng} / \mathrm{ml}$ ) and for VGLUT3 (at a concentration of $400 \mathrm{ng} / \mathrm{ml}$ ). Sections were immersed in this solution overnight at $55^{\circ} \mathrm{C}$. After stringency washes in $4 \times$ standard sodium citrate (SSC) and immersion in RNase solution $(20 \mu \mathrm{g} / \mathrm{ml}$ RNase A, $0.5 \mathrm{~m}$ sodium chloride, $0.01 \mathrm{~m}$ Tris, $\mathrm{pH} 8.0,0.25 \mathrm{~mm} \mathrm{EDTA}$ ) at $37^{\circ} \mathrm{C}$ for $30 \mathrm{~min}$ and stringency washes in $0.1 \times$ SSC, sections were preincubated once for $30 \mathrm{~min}$ in $0.1 \mathrm{M}$ Tris- $\mathrm{HCl}$ containing 3\% normal goat serum and $0.3 \%$ Triton $\mathrm{X}-100$. The sections were then placed overnight in the same solution containing antidigoxigenin antibodies conjugated to alkaline phosphatase in a dilution of 1:1000 (Roche). The next day, sections were rinsed in $0.1 \mathrm{M}$ Tris$\mathrm{HCl}, \mathrm{pH} 7.5$, followed by rinses in $0.1 \mathrm{M}$ Tris, $\mathrm{pH}$ 9.5. The color reaction was elicited by immersing the sections in alkaline phosphatase substrate solution $(0.1 \mathrm{~m}$ Tris, $\mathrm{pH} 9.5,0.45 \mathrm{~mm}$ nitro blue tretrazolium, $0.45 \mathrm{~mm} 5$-bromo-4-chloro-3-indolyl 10 phosphate) for 3-4 h. Sections were mounted on gelatin-coated slides, air dried, and coverslipped under Vectamount (Vector Laboratories, Burlingame, CA).

Immunohistochemistry. All steps were performed under gentle agitation on a horizontal rotator (Lab-Line; Fisher Scientific, Pittsburgh, PA). Entire series of free-floating sections were rinsed in $0.1 \mathrm{M} \mathrm{PB}, \mathrm{pH}$ 7.4, placed into $1 \%$ sodium borohydride for $15 \mathrm{~min}$, thoroughly rinsed in 0.1 M PB again, pretreated with 0.1 м PB containing $0.2 \%$ Triton X-100, and transferred into a solution containing mouse anti-tyrosine hydroxylase (1:10,000; ImmunoStar, Hudson, WI), rabbit anti-neuronal nitric oxide synthase (1:8000; Sigma), or rabbit anti-substance P (1:5000; Immunostar) in $0.1 \mathrm{M} \mathrm{PB}$ with $0.2 \%$ Triton X-100. The following day, after thorough rinsing in $0.1 \mathrm{M} \mathrm{PB}$, sections were placed for $1 \mathrm{~h}$ in $0.1 \mathrm{M} \mathrm{PB}$ containing $0.2 \%$ Triton $\mathrm{X}-100$ and a biotinylated antibody against mouse or rabbit IgGs (Vector Laboratories) accordingly, at a dilution of 1:200. The sections were again rinsed in $0.1 \mathrm{M} \mathrm{PB}$ with $0.2 \%$ Triton $\mathrm{X}-100$ and immersed for another hour in $0.1 \mathrm{M} \mathrm{PB}$ containing $0.2 \%$ Triton $\mathrm{X}-100$ and avidin-biotin peroxidase complex (ABC; 1:200; Vector Laboratories). After thorough rinsing in $0.1 \mathrm{M} \mathrm{PB}$, a color reaction was elicited by immersing the sections for $\sim 15 \mathrm{~min}$ in $0.05 \mathrm{M} \mathrm{PB}$ containing $0.05 \%$ 3,3'-diaminobenzidine, $0.04 \%$ ammonium chloride, $0.2 \% \beta$-D-glucose, and $0.0004 \%$ glucose oxidase. Reacted sections were mounted onto gelatin-coated slides, dehydrated in ascending concentrations of ethyl alcohol, transferred into xylene, and coverslipped with Permount (Fisher Scientific). No staining was observed when the primary or secondary antibodies or $\mathrm{ABC}$ reagents were omitted.

Nissl stain. Sections were mounted onto gelatin-coated slides, air dried, dehydrated and rehydrated through graded concentrations of ethyl alcohol, put into distilled water for $2 \mathrm{~min}$, transferred into cresyl violet $(0.2 \%$ cresyl violet acetate, $20 \mathrm{~mm}$ acetic acid buffer, $\mathrm{pH} 4.0)$, and
Table 1. Overview of experiments

\begin{tabular}{llll}
\hline Case & VGLUT1 & VGLUT2 & VGLUT3 \\
\hline 05179 & $\mathrm{x}$ & $\mathrm{x}$ & \\
05216 & $\mathrm{x}$ & + & $\mathrm{x}$ \\
06054 & $\mathrm{x}$ & $\mathrm{x}$ & $\mathrm{x}$ \\
06056 & $\mathrm{x}$ & \\
06084 & + & + & $\mathrm{x}$ \\
06085 & & + & $\mathrm{x}$ \\
06150 & & + & $\mathrm{x}$ \\
06151 & & + & \\
06152 & & &
\end{tabular}

The $x$ symbol indicates that a complete series of VGLUT-VTA-projecting neurons was plotted and counted. The + symbol indicates that a partial series of VGLUT-VTA-projecting neurons was plotted and counted to provide additional details about specific structures, such as, for example, ventral pallidum and lateral hypothalamic area (VGLUT2) and dorsal peduncular cortex (VGLUT1).

left there for $30 \mathrm{~min}$. Another dehydration through graded alcohols preceded transfer of the sections into xylene and coverslipping with Permount (Fisher Scientific).

Analysis. With the aid of a Nikon (Tokyo, Japan) Optiplot light microscope linked by stage position transducers to a dedicated hardwaresoftware platform (MDplot; Accustage, Shoreview, MN), outlines and major landmarks of interest were drawn using a $4 \times$ objective. Then, with the $20 \times$ objective and aid of the same hardware-software platform, double-labeled and retrogradely labeled neurons were plotted in every other section in series of equidistantly spaced, rostrocaudally ordered sections through the entire brain. In addition, in two series, VGLUT2 mRNA-positive, VTA-projecting neurons were plotted in every section of a series of equidistant, rostrocaudally ordered sections. An aggregation of at least eight gold-silver particles was required to identify singlelabeled VTA-projecting neurons. This disallowed inclusion in the anal- 

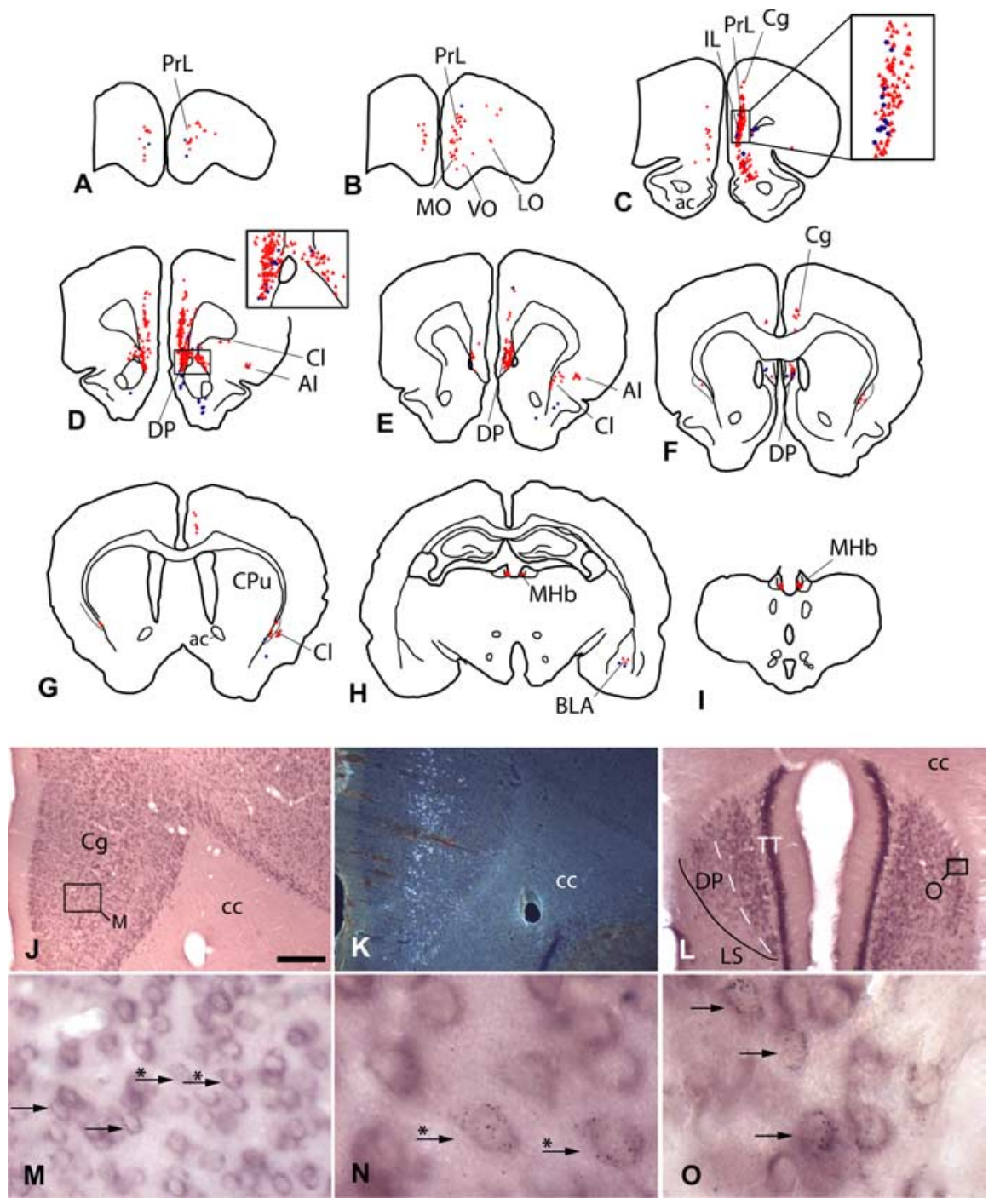

Figure 3. $A-I$, Schematic representations showing the distribution of VGLUT1 mRNA-positive, VTA-projecting neurons (red triangles) and retrogradely labeled neurons lacking detectable VGLUT1 mRNA (blue dots). Each triangle or dot reflects one neuron. Note that few retrogradely labeled neurons were not positive for VGLUT1 mRNA (see also insets in C, D). Most double-labeled neurons were situated in the medial prefrontal cortex [e.g., in the prelimbic (PrL) and infralimbic (IL) cortices (D-E)]. To simplify the diagram, retrogradely labeled neurons were plotted only in the areas expressing VGLUT1 mRNA-positive neurons. [For complete retrograde labeling after tracer injections into the VTA, see Geisler and Zahm $(2005,2006 \mathrm{~b})]$. J-0, VGLUT1mRNA was abundantly expressed by neurons in the cingulate cortex $(\boldsymbol{J})$, an area that also contained numerous VTA-projecting neurons ( $\boldsymbol{K}$, shown in dark field). At higher magnifications $[\boldsymbol{M}, \boldsymbol{N}$ (an enlargement of $\boldsymbol{M}$ )], the retrograde labeling (black puncta) can be clearly recognized in neurons positive for VGLUT1 mRNA (purple). The dorsal peduncular cortex $(\boldsymbol{E}, \boldsymbol{F}, \boldsymbol{L})$, situated lateral to the taenia tecta (TT) and dorsomedial to the lateral septum (LS), also contained many double-labeled neurons ( $\boldsymbol{O}$, an enlargement of $\boldsymbol{L}$ ). Arrows indicate double-labeled neurons; arrows with asterisks indicate the same double-labeled neurons in $\boldsymbol{M}$ and $\boldsymbol{N}$. DP, Dorsal peduncular cortex; Al, agranular insular cortex; ac, anterior commissure; BLA, basolateral amygdala; $c$, corpus callosum; $\mathrm{Cg}$, cingulate cortex; $\mathrm{Cl}$, claustrum; $\mathrm{CPu}$, caudate-putamen; $\mathrm{LO}$, lateral orbital cortex; $\mathrm{MHb}$, medial habenula; $\mathrm{MO}$, medial orbital cortex; V0, ventral orbital cortex. Scale bar: (in $J) ~ J, K, 300 \mu \mathrm{m} ; \boldsymbol{L}, 200 \mu \mathrm{m} ; \boldsymbol{M}, 30 \mu \mathrm{m} ; \boldsymbol{N}, \mathbf{0}, 10 \mu \mathrm{m}$.

ysis of dendrites separated from a cell body by sectioning, which is important because (1) probe labeling would not be present in dendrites, which frequently exhibited a grain or two, and (2) it prevented from counting the same neuron twice (i.e., because of grains in the cell body and, at some distance, in a dendrite). The same number of gold-silver particles was used for the identification of double-labeled neurons. Before neurons were recorded as double labeled, care was taken to ascertain that the gold-silver particles were indeed within VGLUT mRNA-positive neurons, rather than below or above, by slowly focusing through them. Brain areas and nuclei were delineated using standard cytoarchitectonic criteria (Paxinos and Watson, 1998; Hur and Zaborszky, 2005). If the VGLUT in situ hybridization signal alone provided inadequate informa- tion to delineate brain areas/nuclei of interest, drawings obtained from adjacent sections, which either were stained with cresyl violet (e.g., to delineate hypothalamic and brainstem nuclei) or were immunolabeled with rabbit anti-neuronal nitric oxide synthase (to delineate forebrain areas and laterodorsal and pedunculopontine tegmental nuclei) or rabbit anti-substance $\mathrm{P}$ (to delineate basal forebrain areas), were superimposed onto the corresponding maps of VGLUT-VTA-projecting neurons (see Figs. 6, 7). Double-labeled neurons in delineated brain areas were counted in series of equidistantly spaced (500 $\mu \mathrm{m}$ apart), rostrocaudally ordered series of sections using MDplot (Accustage). For each case, the contribution of double-labeled neurons in each structure (ipsilateral and contralateral) was calculated relative to the sum of all double-labeled neurons in the case. These numbers, in turn, were used to calculate group means and SEM Double-labeled and retrogradely labeled neurons in delineated structures were counted with the aid of MDplot (Accustage), and the values were used to calculate double labeling as a percentage of retrograde labeling, which then in turn were used to calculate the group means \pm SEM for each structure. Maps were arranged and finished with the aid of Adobe Illustrator 11CS (Adobe Systems, Mountain View, CA). Images for illustration were acquired with a digital camera (QIcam Fast 1394; QImaging, Burnaby, British Columbia, Canada), and minor adjustments of contrast were made with the aid of Adobe Photoshop 7 (Adobe Systems).

\section{Results}

\section{Distribution of VGLUT probes}

The mRNAs of the three evaluated VGLUTs were distributed in a minimally overlapping manner in distinct areas of the brain.

VGLUT1 mRNA was expressed in all layers of the cerebral cortex (Fig. $1 A$ ), the dorsal and ventral endopiriform areas, hippocampal formation, medial habenula, basolateral and cortical amygdaloid nuclei, lateral nucleus of the olfactory tract, some thalamic nuclei, some nuclei of the brainstem (including the mesencephalic and principle sensory nuclei of the trigeminal nerve, pontine nuclei, and reticulotegmental field of the pons), and in the granule cell layer of the cerebellum.

Forebrain VGLUT2 mRNA was detected in the ventral endopiriform area; piriform cortex; claustrum; medial, lateral, and posterior septum; diagonal band of Broca; ventral pallidum; anteroventral bed nucleus of the stria terminalis; medial and ventral globus pallidus; medial and lateral preoptic area; subcomissural substantia innominata; caudal bed nucleus of stria terminalis; some nuclei in the medial hypothalamus; lateral hypothalamic area (Fig. $1 B$ ); entopeduncular nucleus (Fig. $1 B$ ); medial and cortical amygdaloid nuclei; all thalamic nuclei, except the reticular nucleus (Fig. $1 B)$; and lateral and medial habenula. In addition, VGLUT2 mRNA was observed in many structures in the brainstem, includ- 
ing the periaqueductal and central gray, medial and lateral geniculate nuclei, superior and inferior colliculus, red nucleus, deep field of the mesencephalic reticular formation, caudal field of the pontine reticular formation, pedunculopontine and laterodorsal tegmental nuclei, cuneiform nucleus, and parabrachial nucleus. Lesser numbers of VGLUT2 mRNA-positive neurons were found in the VTA, interpeduncular nucleus, lateral part of substantia nigra, retrorubral field, and median raphe, and a few VGLUT2 mRNA-positive neurons were detected in the oral field of the pontine reticular formation.

Numerous VGLUT3 mRNA-positive neurons were present in the dorsal and median raphe. VGLUT3 mRNA was also expressed by scattered neurons in the caudate-putamen, accumbens (Fig. 1C), olfactory tubercle (Fig. 1C), ventral pallidum, globus pallidus, hippocampus, lateral habenula, cortex, anterior medial nucleus of the bed nucleus of stria terminalis, medial septum/diagonal band complex, central gray, and medulla oblongata.

Invariably, labeling was completely absent in sections processed with sense probes for VGLUT1, VGLUT2, and VGLUT3 at concentrations that produced specific labeling with antisense probes.

\section{Tracer injections}

Typical WGA-apo HRP-gold injection sites contained irregularly shaped, homogeneous black deposits of silver-enhanced gold surrounded by less dense halos of enhancement product (Fig. 2), which most probably contributed to tracer uptake and transport, because small deposits resulted in considerable numbers of retrogradely labeleled neurons throughout the brain. Injection sites typically were centered on the parabrachial pigmented or paranigral nuclei and extended throughout the rostrocaudal extent of the VTA (Fig. 2C, $C^{\prime}$ ). Retrograde labeling was observed in the same brain structures as described recently (Geisler and Zahm, 2005, 2006a,b). Control injections dorsal and lateral to the VTA produced labeling in structures distinct from those projecting to the VTA. Such control injections, but with Fluoro-Gold rather than WGA-apo HRP-gold as the tracer, were described in detail and illustrated by Geisler and Zahm (2005). For an overview of the experiments, see Table 1.

\section{VGLUT mRNA-positive, VTA-projecting neurons}

\section{VGLUT1}

The majority of VGLUT1 mRNA-positive (VGLUT1), VTAprojecting neurons were in the deep layers of the medial prefrontal cortex (Fig. 3). Here, densely packed, double-labeled neurons occupied the prelimbic, medial orbital, and infralimbic cortices (Fig. $3 A-D$ ) and extended ventralward into an area shown in the atlas of Paxinos and Watson (1998) as corresponding to the dorsal peduncular cortex (Fig. 3D-F,L,O). This band of doublelabeled neurons could be followed caudalward into the cingulate cortex (cingulates 1 and 2), where the numbers of VGLUT1VTA-projecting neurons decreased (Fig. $3 E-G, J, M$ ). In addition, some double-labeled neurons were observed in the ventral orbital, lateral orbital, and agranular insular cortices, as well as in the claustrum/dorsal endopiriform nucleus (Fig. 3B-G). When the numbers of prefrontocortical double-labeled neurons were compared (Table 2), the prelimbic cortex possessed the greatest accumulation $(\sim 43 \%)$, followed by the dorsal peduncular, infralimbic, cingulate, and medial orbital cortices, which each comprised $\sim 5-10 \%$ of total prefrontocortical VGLUT1-VTAprojecting neurons (Table 2). More than $90 \%$ of the retrogradely labeled neurons in the prefrontal cortex were double labeled (Table 2).

Further caudally, an occasional double-labeled neuron was detected in the basolateral nucleus of the amygdala (Fig. $3 H$ ). The only noncortical VGLUT1 mRNA-positive neurons observed to contain retrograde labeling after tracer injections into the VTA were situated in the medial habenula (Fig. 3I). No VGLUT1VTA-projecting neurons were detected in the brainstem.

\section{VGLUT2}

VGLUT2 mRNA-positive (VGLUT2), VTA-projecting neurons were detected in almost all subcortical structures that project to the VTA. Notable exceptions include the accumbens, lateral septum, and dorsal raphe.

Ventral endopiriform area and claustrum. Rostrally, doublelabeled neurons were situated ventral and lateral to the rostral pole of the accumbens in the ventral endopiriform area. From the level of the anterior olfactory nucleus rostrally to the amygdaloid area caudally, the ventral endopiriform area contained few, scattered, strongly positive VGLUT2 mRNA-positive neurons (Fig. $4 A I, A I I)$. A few of these were also retrogradely labeled, and the accumulations of silver grains in some of them were among the densest observed in this study (Fig. 4AIII). This mostly ipsilateral projection accounted for $\sim 1 \%$ of all VGLUT2-VTA-projecting neurons (Table 3).

Densely packed VGLUT2 mRNA-positive neurons in the claustrum exhibited a weaker hybridization signal than did those in the ventral endopiriform area. Few claustral VGLUT2 mRNApositive neurons exhibited retrograde labeling (Fig. 4C).

Septum. Scattered, strongly VGLUT2 mRNA-positive neurons and numerous retrogradely labeled neurons were distributed throughout the entire extent of the medial septum/diagonal band complex. Double-labeled neurons were observed predominantly ipsilateral to the injection and constituted $\sim 2.5 \%$ of all VGLUT2-VTA-projecting neurons (Fig. 4B, C,E; Table 3). The density of VGLUT2 mRNA-positive neurons increased in the 

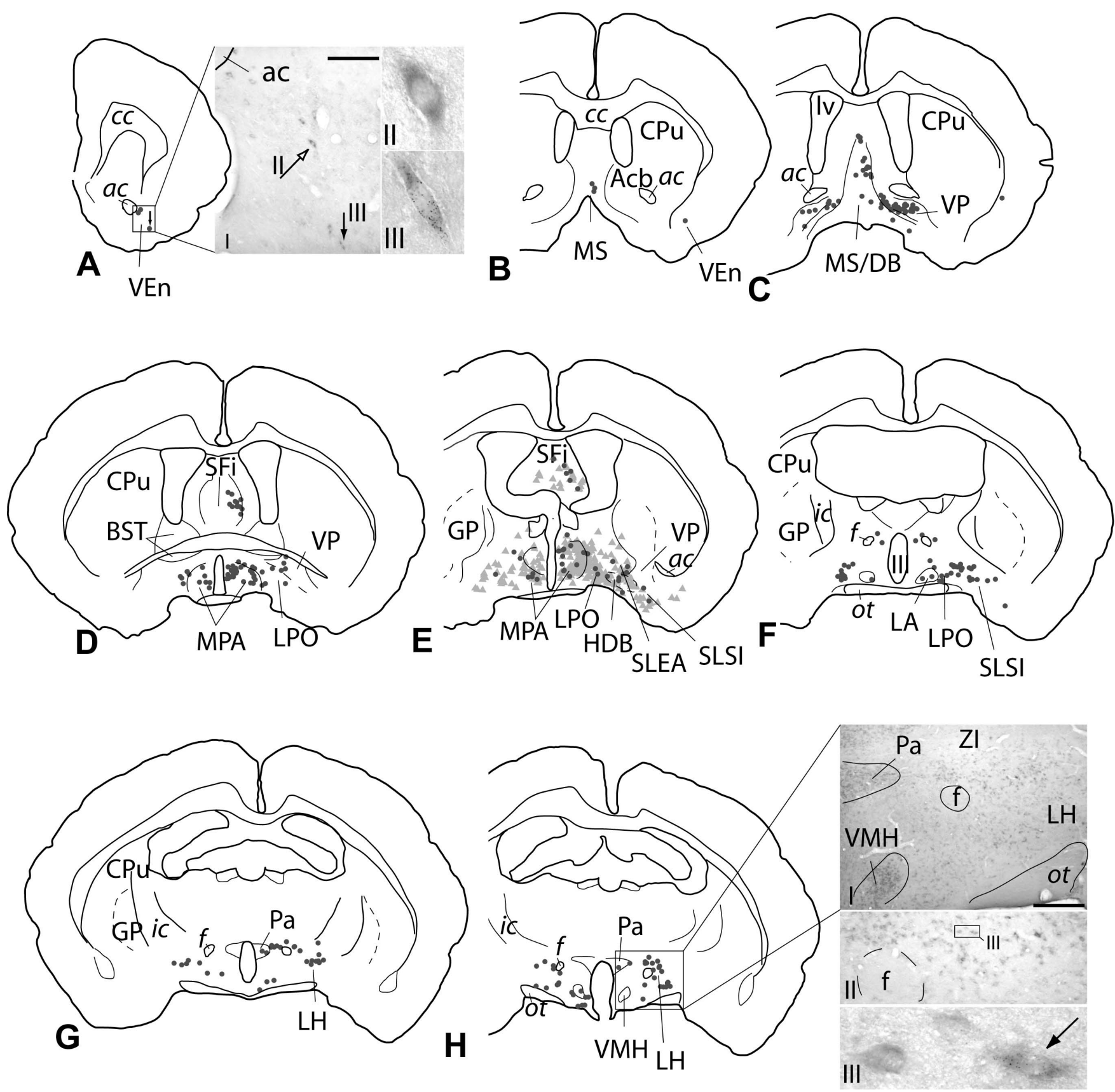

Figure 4. Schematic representations and photomicrographs illustrating the distribution of VGLUT2 mRNA-positive, VTA-projecting neurons in the forebrain. $\boldsymbol{A}-\boldsymbol{H}$, Each dark gray dot represents one double-labeled neuron. The parts of the sections illustrated by photomicrographs are indicated by rectangles on the corresponding drawings. The injection site is illustrated in Figures 2 and $6 F-H$ (case 06151). To give an impression of double labeling relative to neurons exhibiting only retrograde label, retrogradely labeled, probe-negative neurons are shown in $\boldsymbol{E}$ (light gray triangles). VGLUT2 mRNA-positive, VTA-projecting neurons were widely distributed throughout the brain. Relatively few neurons per structure were detected, however. Al, AII, Strongly VGLUT2 mRNA-positive neurons were scattered throughout the ventral endopiriform area. The accumulation of silver grains in some of these neurons was among the densest observed in this study (compare AIII with $H$ HIII, Fig. $6 \mathrm{DII}, \mathrm{DIII}, \mathrm{KII}, \mathrm{KIII})$. $\boldsymbol{H}$, Density and staining intensity of VGLUT2 mRNA-positive neurons in the medial (e.g., paraventricular and ventromedial hypothalamic nucleus) and lateral hypothalamus (HII is an enlargement of $\boldsymbol{H I}$. The fornix serves as a reference mark). An example of a double-labeled neuron is given in $\boldsymbol{H I I I}$ (arrow, enlargement of $\boldsymbol{H I I )}$ ). Black arrows in $\boldsymbol{A}$ and $\boldsymbol{H}$ point to double-labeled neurons. The open arrow in AI points to the VGLUT2 mRNA-positive neuron lacking the retrograde label shown in All. ac, Anterior commissure; Acb, accumbens; BST, bed nucleus of stria terminalis; CPu, caudate-putamen; cc, corpus callosum; DB, diagonal band of Broca; f, fornix; GP, globus pallidus; HDB, horizontal limb of DB; ic, internal capsule; LA, lateroanterior hypothalamic nucleus; LH, lateral hypothalamic area; LPO, lateral preoptic area; Iv, lateral ventricle; MPA, medial preoptic area; MS, medial septum; ot, optic tract; Pa, paraventricular hypothalamic nucleus; SLEA, sublenticular extended amygdala; SLSI, sublenticular substantia innominata; SFi, septofimbrial nucleus; VEn, ventral endopiriform nucleus; VMH, ventromedial hypothalamic nucleus; VP, ventral pallidum; ZI, zona incerta; III, third ventricle. Scale bars: AI, HII, $200 \mu \mathrm{m} ; \boldsymbol{H I}, 300 \mu \mathrm{m} ;$ AII, AIII, HIII, $20 \mu \mathrm{m}$.

septofimbrial nucleus, whereas the intensity of the in situ hybridization signal in individual neurons decreased. Retrograde labeling was observed in some of those, accounting for $\sim 2 \%$ of all VGLUT2-VTA-projecting neurons (Fig. 4D,E; Table 3). Although the lateral septum possessed the greatest density of retro- gradely labeled neurons in the septal area after tracer injections into the VTA and a few lightly stained VGLUT2 mRNA-positive neurons, no double-labeled neurons were detected.

Ventral pallidum. Numerous VGLUT2 mRNA-positive neurons were observed in the ventral pallidum (Fig. 5), which tended 
Table 3. Distribution of VGLUT2 mRNA-positive, VTA-projecting neurons

\begin{tabular}{|c|c|c|}
\hline \multirow[b]{2}{*}{ Structure } & \multicolumn{2}{|c|}{ Percentage of total } \\
\hline & Ipsilateral & Contralateral \\
\hline Lateral hypothalamic area & $10.3 \pm 1.1$ & $3.6 \pm 0.9$ \\
\hline Lateral preoptic area & $6.7 \pm 1.9$ & $1.5 \pm 0.4$ \\
\hline Mesopontine central gray ${ }^{a}$ & $6.6 \pm 2.5$ & $2.2 \pm 0.8$ \\
\hline Medial hypothalamus ${ }^{b}$ & $6.1 \pm 0.7$ & $2.2 \pm 0.7$ \\
\hline Ventral pallidum & $5.3 \pm 1.4$ & $1.6 \pm 0.4$ \\
\hline Mesopontine reticular formation ${ }^{c}$ & $5.0 \pm 0.6$ & $4.3 \pm 0.9$ \\
\hline Lateral habenula & $4.8 \pm 1.0$ & $2.0 \pm 0.7$ \\
\hline Pedunculopontine and laterodorsal tegmental nuclei & $4.0 \pm 0.5$ & $2.5 \pm 0.1$ \\
\hline Medial preoptic area & $3.5 \pm 0.8$ & $1.1 \pm 0.1$ \\
\hline Parabrachial nucleus & $3.5 \pm 0.6$ & $1.6 \pm 1.1$ \\
\hline Median raphe & $3.1 \pm 0.7$ & NA \\
\hline Cuneiform nucleus & $2.5 \pm 0.4$ & $2.0 \pm 0.7$ \\
\hline Medial septum/diagonal band & $2.3 \pm 0.5$ & $0.3 \pm 0.1$ \\
\hline Septofimbrial nucleus & $1.8 \pm 0.7$ & $0.1 \pm 0.1$ \\
\hline Basal forebrain $^{d}$ & $1.7 \pm 0.2$ & $0.3 \pm 0.1$ \\
\hline Ventral endopiriform area & $0.8 \pm 0.1$ & 0 \\
\hline 0thers ${ }^{e}$ & $4.0 \pm 0.7$ & $2.3 \pm 0.5$ \\
\hline
\end{tabular}

Values are percentages of total VGLUT2-VTA-projecting neurons and are listed in descending order; $n=4$ rats ( 617 811,873 , and 1014 double-labeled neurons per series of sections, respectively, were plotted). NA, Not applicable. ${ }^{a}$ Includes periaqueductal gray and pontine central gray.

${ }^{b}$ Includes anterior hypothalamic area; paraventricular, ventromedial, and posterior hypothalamic nuclei; tuber cinereum; and dorsal hypothalamic area.

Includes deep field of mesencephalic reticular formation and oral and caudal field of pontine reticular formation. ${ }^{d}$ Includes subcommissural and sublenticular extended amygdala and sublenticular substantia innominata.

${ }^{e}$ Includes claustrum, anteroventral bed nucleus of the stria terminalis, ventral tier of zona incerta, parafascicular thalamic nucleus, supramammillary nucleus, and superior colliculus.

to be densely packed and lightly VGLUT2 mRNA positive (Fig. $5 B, G)$ rostromedially and more loosely arranged and strongly VGLUT2 mRNA positive rostrolaterally and caudally (Fig. $5 B, H, I$ ). Similar to the retrograde labeling (Fig. $5 C, F$ ), numerous double-labeled neurons occupied the rostral levels of the ventral pallidum (Figs. 4C, 5C,G,H), whereas at its caudal levels, fewer were observed (Figs. $4 D, E, 5 F, I$ ). Of the retrogradely labeled neurons in the ventral pallidum, $35.0 \pm 4.3 \%$ ( $n=5$ rats) were VGLUT2 mRNA positive. Double-labeled neurons in the ventral pallidum comprised $\sim 7 \%$ of all VGLUT2-VTAprojecting neurons (Table 3 ).

Preoptic region and adjacent structures. Between the scattered and strongly VGLUT2 mRNA-positive neurons of the ventral pallidum and those densely packed in the medial preoptic area, loosely arranged VGLUT2 mRNA-positive neurons were situated in the lateral preoptic area (Fig. 5E). These increased in number and staining intensity in the transition to the lateral hypothalamic area. Approximately $8.5 \%$ of all VGLUT2-VTAprojecting neurons were located bilaterally in the lateral preoptic area (Fig. $4 D-F$ ). In one case, we determined that $24.0 \%$ of the retrogradely labeled neurons were double labeled. Numerous VGLUT2-VTA-projecting neurons were also found bilaterally in the medial preoptic area, accounting for $\sim 4.5 \%$ of all VGLUT2VTA-projecting neurons (Figs. 4D-F, 5E, F; Table 3). Only an occasional double-labeled neuron was situated in the anteroventral bed nucleus of the stria terminalis, subcommissural and sublenticular extended amygdala, and sublenticular substantia innominata (Figs. $4 E, F, 5 B, C$; Table 3 ).

Hypothalamus. With 15\% of all VGLUT2-VTA projecting neurons, the lateral hypothalamic area possessed the largest accumulation of VGLUT2-VTA-projecting neurons (Table 3). These were present throughout its entire rostrocaudal extent on both sides of the brain but were more prominent ipsilateral to the injection (Figs. 4G, H, 6A-E; Table 3). Of the retrogradely labeled neurons in the lateral hypothalamic area, $22.6 \pm 4.3 \%(n=5$ rats) were double labeled. A moderate number of double-labeled neurons was observed in nuclei of the medial hypothalamus (i.e., in the ventromedial and posterior hypothalamic nuclei), as were a few in each of the anterior and dorsal hypothalamic areas, the lateroanterior and paraventricular hypothalamic nuclei, and the tuber cinereum (Figs. 4G,H, 6A-E).

Lateral habenula and fasciculus retroflexus. Many VGLUT2 mRNA-positive (Fig. 6DI) and retrogradely labeled neurons were situated in the lateral habenula. A moderate number of lateral habenular neurons contained both markers (Fig. 6B-D). These represented $\sim 7 \%$ of all VGLUT2-VTA-projecting neurons (Table 3). Caudal to the lateral habenula, double-labeled neurons were observed in the vicinity of the fasciculus retroflexus (the main efferent fiber bundle of the habenula) occupying the parafascicular thalamic nucleus and adjacent reticular formation (Fig. 6E).

Median raphe. In contrast to the dorsal raphe, where VGLUT2 mRNA-positive neurons were not detected, the median raphe contained scattered, darkly stained VGLUT2 mRNA-positive neurons (Fig. $6 \mathrm{~K}$ ), some of which were retrogradely labeled (Fig. $6 J-L$ ), accounting for $\sim 3 \%$ of all VGLUT2-VTA-projecting neurons. Of the retrogradely labeled neurons in the median raphe, $18.7 \pm 1.8 \%$ ( $n=3$ rats) were VGLUT2 mRNA positive.

Pedunculopontine tegmental nucleus and adjacent structures. A cluster of VGLUT2 mRNA-positive neurons, some of which contained retrograde labeling, stretched from ventrolateral at the level of the caudal red nucleus to dorsomedial at the level of the laterodorsal tegmental nucleus (Fig. 6I-M). Although rostrally this cluster of neurons was distinct from the surrounding deep field of the mesencephalic reticular formation because of the generally low VGLUT2 mRNA expression there (Fig. 7A), at the level of the laterodorsal tegmental nucleus, VGLUT2 mRNA-positive and double-labeled neurons were widely and seemingly diffusely distributed throughout the reticular formation (Fig. $7 B, D$ ). Only after superimposing plots of double-labeled neurons onto adjacent sections immunoreacted to exhibit neuronal nitric oxide synthase (Fig. $7 A-C$ ) could it be concluded that a moderate number of the double-labeled neurons in this elongated cluster were located in the pedunculopontine and laterodorsal tegmental nuclei (Fig. 6I-M). This bilateral projection accounted for $\sim 6.5 \%$ of all VGLUT2-VTA-projecting neurons (Table 3). On both sides of the brain, many double-labeled neurons were also observed in the cuneiform nuclei (Fig. $6 K, L$; Table 3 ), periaqueductal gray and parabrachial nuclei (Fig. $6 I-M$, Table 3), as well as in the deep field of the mesencephalic reticular formation and oral field of the pontine reticular formation (Fig. 6I-M, Table 3).

\section{VGLUT3}

Most VGLUT3 mRNA-positive, VTA-projecting neurons (VGLUT3-VTA-projecting neurons) were located in the dorsal $(\sim 73 \%)$ and median $(\sim 20 \%)$ raphe (Fig. 8, Table 4$)$. Although the median raphe contained many VGLUT3 mRNA-positive neurons, only $\sim 20 \%$ of the retrogradely labeled neurons were positive for VGLUT3 mRNA (Figs. 8D-G, 9; Table 4). In contrast, in the dorsal raphe, where the VGLUT3 hybridization signal was weaker, $\sim 65 \%$ of retrogradely labeled neurons were positive for VGLUT3 mRNA (Fig. $8 D-G$, Table 4).

Scattered VGLUT3 mRNA-positive neurons were observed in the lateral habenula, of which very few were retrogradely labeled (Fig. $8 \mathrm{~B}$ ). An occasional double-labeled neuron was observed in the medial division of the bed nucleus of the stria terminalis (Fig. $8 A$ ). Caudally, the central gray of the pons and medulla oblon- 


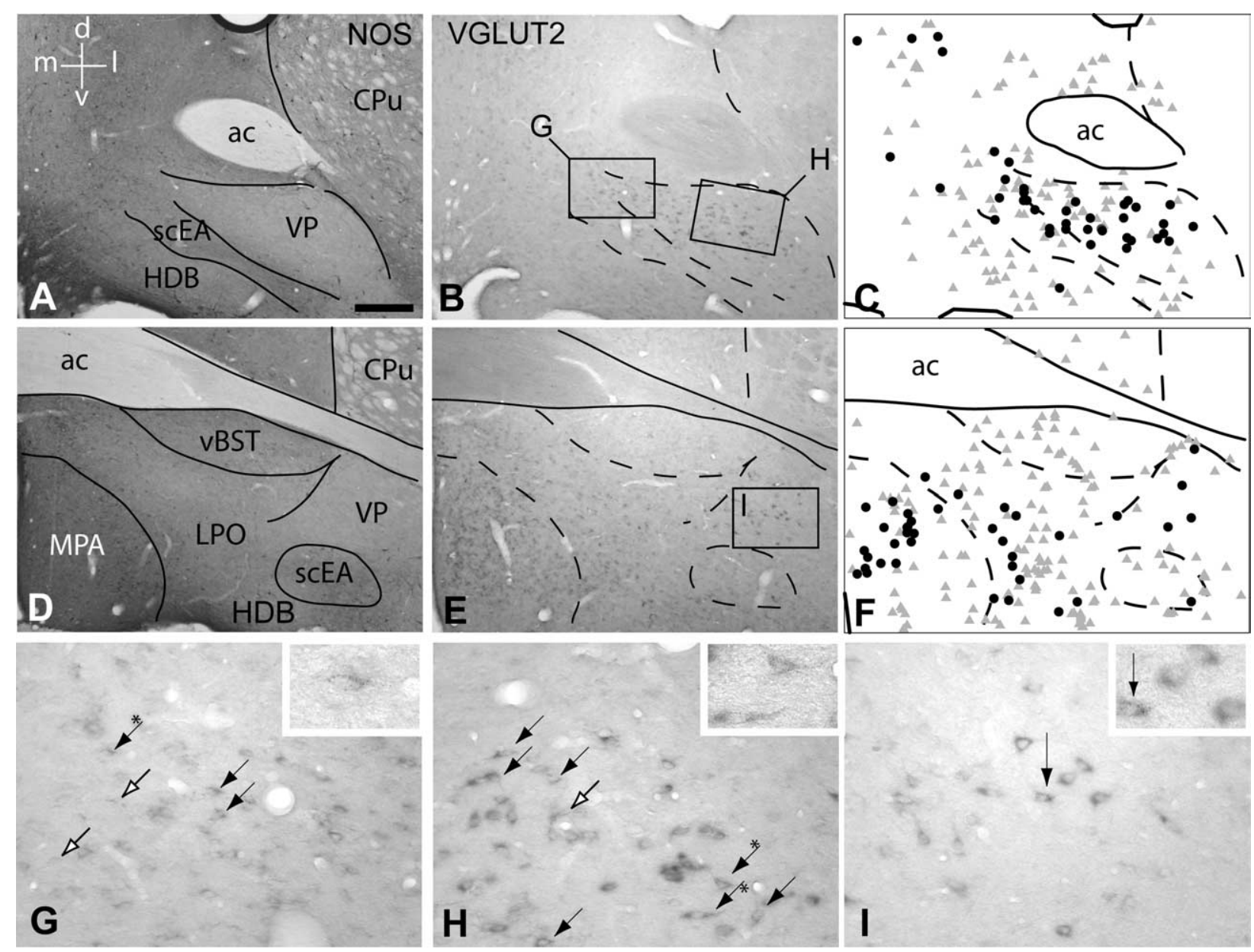

Figure 5. VGLUT2 mRNA-positive, VTA-projecting neurons in the ventral pallidum (VP). $\boldsymbol{A}-\mathbf{C}$ and $\boldsymbol{D}-\boldsymbol{F}$ illustrate rostral and caudal parts of the VP, respectively. $\boldsymbol{A}$ and $\boldsymbol{D}$ are micrographs of sections immunoreacted to exhibit neuronal nitric oxide synthase (NOS) to delineate the VP and surrounding structures. $\boldsymbol{B}$ and $\boldsymbol{E}$ illustrate sections adjacent to those shown in $\boldsymbol{A}$ and $\boldsymbol{D}$, respectively, which were subjected to silver enhancement to visualize the retrograde tracer and probe against VGLUT2 mRNA. $\boldsymbol{B}, \boldsymbol{E}, \mathbf{G}-\mathbf{I}$, Numerous densely packed VGLUT2 mRNA-positive neurons were observed in the rostral VP $(\boldsymbol{B}, \boldsymbol{G}, \boldsymbol{H})$, whereas fewer, but darkly stained, VGLUT2 mRNA neurons were observed further caudal in the VP $(\boldsymbol{E}, \boldsymbol{I}) . \boldsymbol{A}-\boldsymbol{F}, \mathrm{VGLUT2}$ mRNA-positive neurons were also present in the subcommissural extended amygdala (scEA), horizontal limb of diagonal band of Broca (HDB), and medial (MPA) and lateral (LPO) preoptic area (D, $\boldsymbol{E})$. C and $\boldsymbol{F}$ are schematic representations of $\boldsymbol{B}$ and $\boldsymbol{E}$, respectively, illustrating retrogradely labeled neurons (gray triangles) and VGLUT2 mRNA-positive, VTA-projecting neurons (black dots). Note that although many VGLUT2 mRNA-positive neurons $(\boldsymbol{B}, \boldsymbol{E})$ and retrogradely labeled neurons (gray triangles) were present in the basal forebrain, relatively few neurons contained both markers (black dots). $\boldsymbol{G}$ and $\boldsymbol{H}$ are enlargements of the boxed areas in $\boldsymbol{B} ; \boldsymbol{I}$ is an enlargement of the boxed area in $\boldsymbol{E}$. Black arrows point to double-labeled neurons. Black arrows with asterisks point to the double-labeled neurons that are enlarged in the insets. Open arrows point to retrogradely labeled neurons. $d$, Dorsal; I, lateral; v, ventral; $m$, medial; ac, anterior commissure; CPu, caudate-putamen; vBST, ventral bed nucleus of stria terminalis. Scale bar: (in A) $A-F, 300 \mu \mathrm{m} ; G-I, 20 \mu \mathrm{m}$.

gata contained some VGLUT3-VTA-projecting neurons (Fig. $8 H)$.

\section{Discussion}

This study reveals that glutamatergic neurons innervating the VTA are present in almost all structures projecting there. Notable exceptions are the accumbens and lateral septum. VGLUT1VTA-projecting neurons are densely packed and located almost exclusively in the prefrontal cortex, mainly in its prelimbic and dorsal peduncular areas. In contrast, VGLUT2-VTA-projecting neurons are scattered throughout most subcortical structures that innervate the VTA. Forebrain areas containing relatively many VGLUT2-VTA-projecting neurons include, in order of descending numbers, the lateral hypothalamic and preoptic areas, medial hypothalamus, ventral pallidum, lateral habenula, medial preoptic area, and medial septum/diagonal band complex. Ascending (brainstem) VGLUT2-VTA-projecting neurons were observed in the periaqueductal and central gray, mesencephalic and pontine reticular formation, pedunculopontine and lat- erodorsal tegmental nuclei, parabrachial and cuneiform nuclei, and median raphe. VGLUT3-VTA-projecting neurons were situated predominantly in the dorsal and median raphe.

An overview of the glutamatergic afferents of the VTA is given in Figure 10. As noted above, whereas VGLUT1- and VGLUT3VTA-projecting neurons are restricted to a few brain areas (prefrontal cortex and mesencephalic raphe nuclei, respectively), VGLUT2-VTA-projecting neurons are situated in many. The values reported in Figure 10 must be regarded as estimations, because they reflect summations of values for VGLUT1-, VGLUT2-, and VGLUT3-VTA-projecting neurons generated in separate groups of rats (Table 1) and do not take into account differences in the detectability of the three probes as a result of differences in the intensity of the in situ hybridization signal, nor possible colocalizations of VGLUT2 and VGLUT3 mRNA in VTA-projecting neurons in the median raphe and lateral habenula. With these caveats in mind, it is nonetheless informative to consider that, in terms of individual structures, the prelimbic 

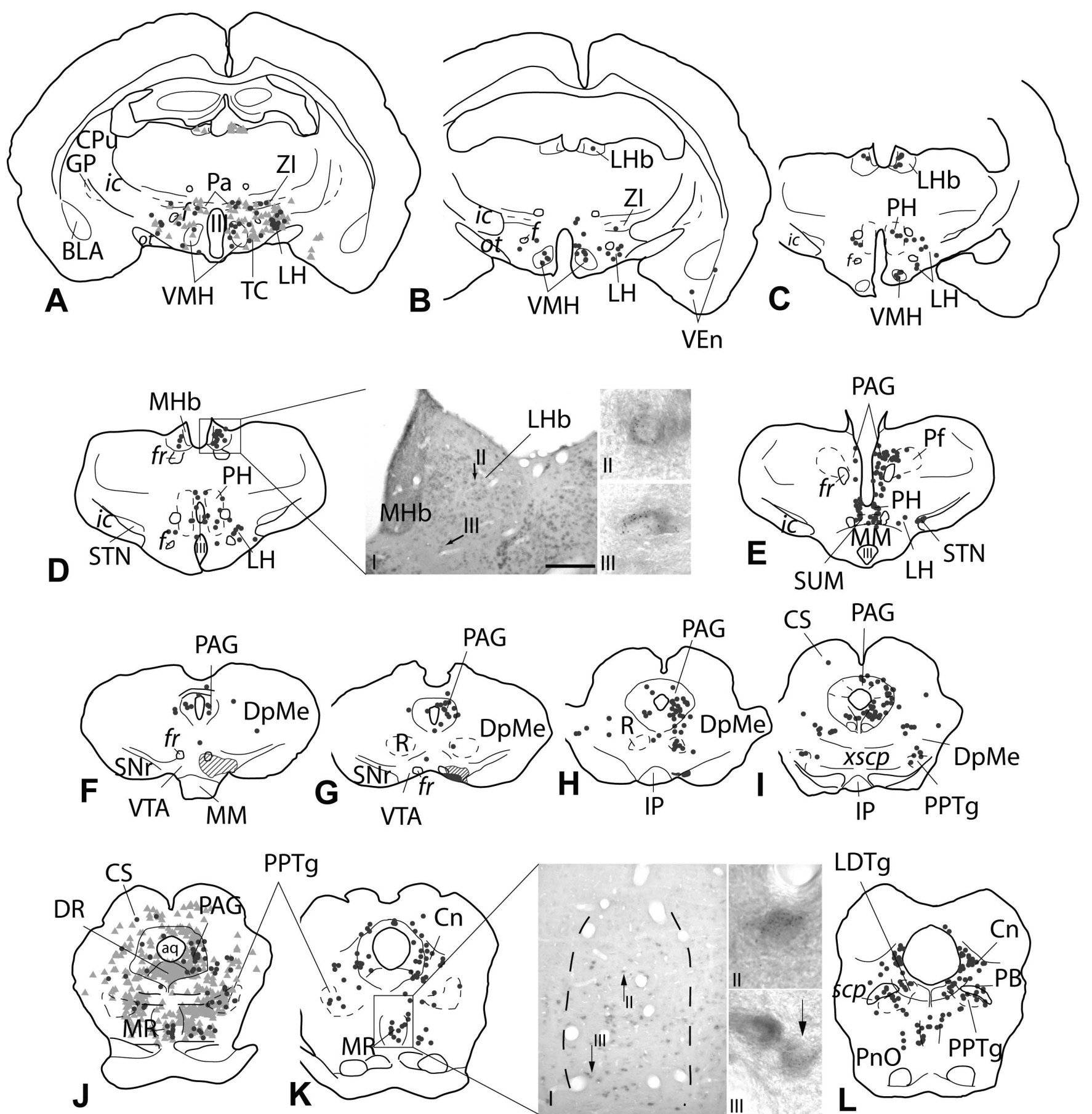

PPTg
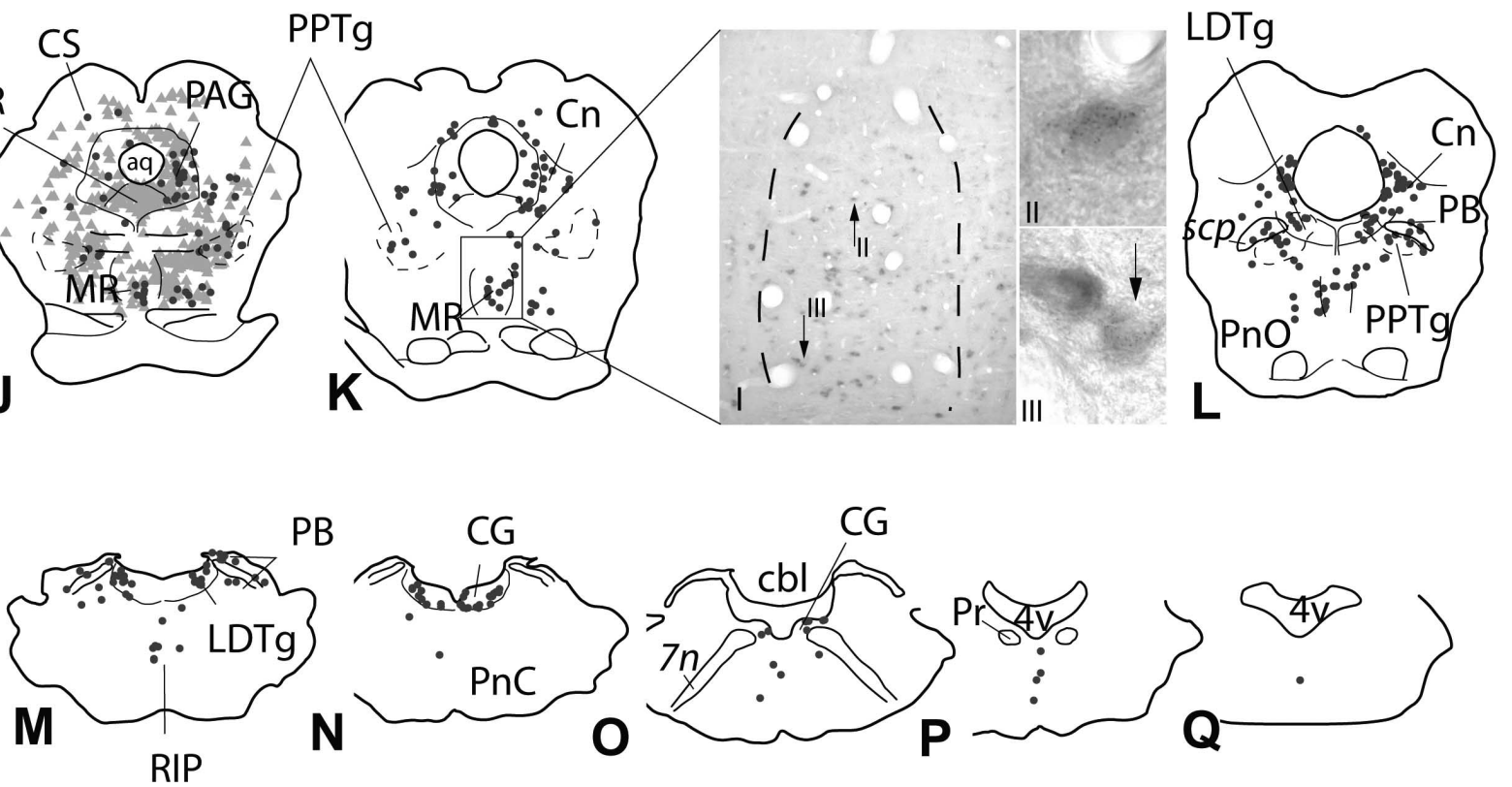

Figure 6. A-Q, Continuation of the series of sections shown in Figure 4 illustrating the distribution of VGLUT2 mRNA-positive, VTA-projecting neurons in the caudal forebrain and brainstem. Symbols and formatting are as in Figure 4. The injection site is illustrated in $\boldsymbol{F}-\boldsymbol{H}$. Note that the center of the injection site $(\boldsymbol{G})$ is surrounded by a less dense halo of silver enhancement product $(\boldsymbol{F}$, $\boldsymbol{G}$, hatched area). $\boldsymbol{A}, \boldsymbol{J}$, To give an impression of double labeling relative to neurons exhibiting only retrograde label, retrogradely labeled, probe-negative neurons are shown (light gray triangles). VGLUT2 mRNA-positive, VTA-projecting neurons were widely distributed throughout the forebrain and brainstem. D, Numerous VGLUT2 mRNA-positive neurons were present in the lateral habenula $(\boldsymbol{D I})$, of which a moderate number contained retrograde labeling (DII, DIII). $\boldsymbol{K}$, The median raphe contained scattered, strongly VGLUT2 mRNA-positive neurons (KI), some of which were retrogradely labeled $(\boldsymbol{K I I}, \boldsymbol{K I I I})$. Black arrows in $\boldsymbol{D}$ and $\boldsymbol{K}$ point to double-labeled neurons. aq, Aqueduct; BLA, basolateral amygdala; cbl, cerebellum; (G, central gray; (Figure legend continues) 

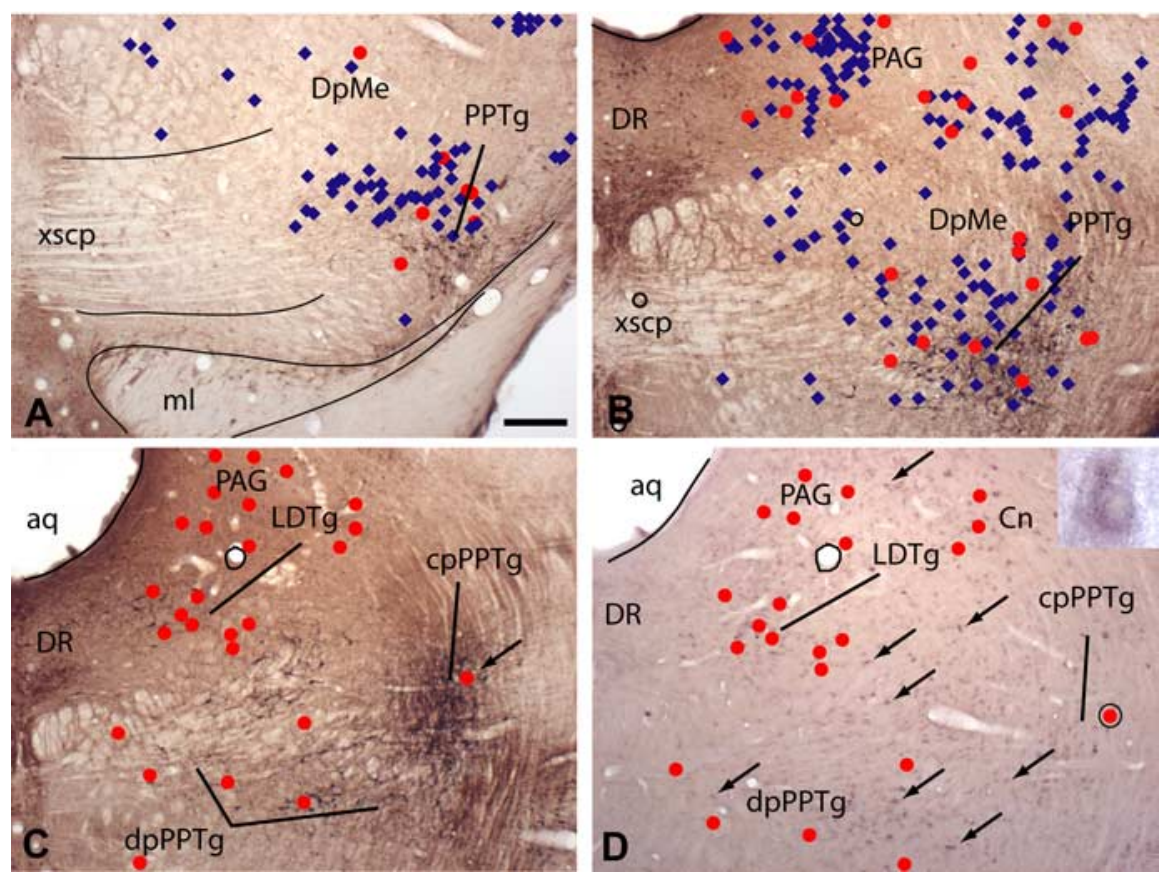

Figure 7. Micrographs illustrating sections through rostral $(\boldsymbol{A})$, middle $(\boldsymbol{B})$, and caudal $(\boldsymbol{C}, \boldsymbol{D})$ levels of the pedunculopontine tegmental nucleus (PPTg). $\boldsymbol{A}-\boldsymbol{C}$, Sections immunoreacted for neuronal nitric oxide synthase, which identifies the PPTg and laterodorsal tegmental nucleus (LDTg). $\boldsymbol{D}$, A section, adjacent to that in $\boldsymbol{C}$, subjected to nonisotopic in situ hybridization against VGLUT2 mRNA and silver intensification. The positions of VGLUT2 mRNA-positive ( $\boldsymbol{A}, \boldsymbol{B}$, blue diamonds) and VGLUT2-VTAprojecting ( $\boldsymbol{A}-\boldsymbol{D}$, red dots) neurons were plotted onto the sections shown in $\boldsymbol{A}-\boldsymbol{C}$ from appropriately processed adjacent sections, such as that shown in $\boldsymbol{D}$ (some VGLUT2 mRNA-positive neurons are indicated by arrows) after tracer injection in the VTA. $\boldsymbol{A}$, Note that relatively few VGLUT2 $m$ RNA-positive neurons are found rostrally and these are located predominantly in the vicinity of the PPTg. $\boldsymbol{B}, \boldsymbol{D}$, In contrast, numerous VGLUT2 mRNA-positive neurons are scattered within the reticular formation more caudally. $\boldsymbol{C}$, Only after having superimposed the plots of double-labeled neurons onto adjacent sections reacted for neuronal nitric oxide synthase immunoreactivity did it become apparent that double-labeled neurons are localized in the dissipated (dp) and compact (cp) parts of the PPTg and in the LDTg, periaqueductal gray (PAG), and cuneiform nucleus ( $(n)$. The encircled red dot in $\boldsymbol{D}$ indicates a double-labeled neuron enlarged in the inset. The same double-labeled neuron, when plotted onto the adjacent section immunoreacted for neuronal nitric oxide synthase, occupies the compact part of the PPTg (C, arrow). Note in $\boldsymbol{D}$ that only a small proportion of VGLUT2 mRNA-positive neurons (some are indicated by arrows) are also retrogradely labeled (red dots). Not shown are retrogradely labeled neurons that do not contain VGLUT2 mRNA. aq, Aqueduct; DpMe, deep field of mesencephalic reticular formation; DR, dorsal raphe; $\mathrm{ml}$, medial lemniscus; xscp, decussation of the superior cerebellar peduncle. Scale bar: (in $\boldsymbol{A}$ ) $\boldsymbol{A}, \boldsymbol{B}, 200$ $\mu \mathrm{m} ; \boldsymbol{C}, \boldsymbol{D}, 250 \mu \mathrm{m}$.

cortex contains the most VGLUT mRNA-positive, VTAprojecting neurons $(\sim 17 \%)$, followed by the lateral hypothalamus $(\sim 7 \%)$, periaqueductal and central gray $(\sim 6 \%)$, and the dorsal raphe $(\sim 5 \%)$.

VGLUT2-VTA-projecting neurons are invariably a minor component of VTA-projecting neurons in structures represented by such neurons. In contrast, projections from the prefrontal cortex are (almost) entirely glutamatergic, which fits the well established association of VTA excitation with stimulation of the prefrontal cortex (Carr and Sesack, 2000, and references therein). A few retrogradely labeled neurons in the prefrontal cortex, however, lacked VGLUT1 mRNA signal. Whether this was because of technical issues or some cortical VTA-projecting neurons using a transmitter other than glutamate (e.g., GABA) awaits further in- vestigation. Interestingly, some identified prefrontal cortical VTA-projecting axons exhibit symmetrical (commonly regarded as inhibitory) synapses in the VTA (Sesack and Pickel, 1992).

We mapped substantial numbers of VGLUT2 mRNA-positive, VTAprojecting neurons in the ventral pallidum (Fig. 5), which is consistent with the recent observation of VGLUT2 mRNA-positive neurons there (Hur and Zaborszky, 2005) and suggests that the well known inhibitory GABAergic (Kalivas et al., 1993) projection from the ventral pallidum to the VTA may be tempered by a significant excitatory component. It will be interesting to learn whether ventral pallidal glutamatergic projections differently target VTA dopaminergic, GABAergic, or glutamatergic neurons or combinations of these. Also in accordance with recent neuroanatomical and electrophysiological studies, we observed VGLUT mRNA-positive, VTAprojecting neurons in the prefrontal cortex (Sesack and Pickel, 1992; Carr and Sesack, 2000), laterodorsal (Omelchenko and Sesack 2005; Lodge and Grace, 2006a) and pedunculopontine (Charara et al., 1996; Floresco et al., 2003; Lodge and Grace, 2006b) tegmental nuclei, and a few in the superior colliculus (Coizet et al., 2003) and anteroventral bed nucleus of the stria terminalis (Georges and AstonJones, 2001, 2002).

\section{Methodological considerations}

The combination of WGA-apo HRP-gold in tracing with nonisotopic in situ hybridization was discussed recently (JongenRelo and Amaral, 2000; Geisler and Zahm, 2006b). Visualization of the tracer by silver enhancement does not interfere with nonisotopic in situ hybridization and results in a punctiform, high-contrast black signal. Because retrogradely labeled neurons typically contained more than 20 gold-silver particles (Fig. 9C), an aggregation of at least eight was regarded as necessary to identify labeled neurons. This strict criterion may have resulted in some false-negative outcomes but prevented counting of neurons more than once (see Materials and Methods). Whereas uptake of tracer into passing axons is always a possibility, the present retrograde labeling closely resembles that observed previously in our laboratory and is in good accord with results from other laboratories (Geisler and Zahm, 2005, 2006b, and references therein). Control injections into the adjacent substantia nigra reticulata produced ret-

\footnotetext{
Cn, cuneiform nucleus; $\mathrm{CPu}$, caudate-putamen; CS, superior colliculus; DpMe, deep field of mesencephalic reticular formation; DR, dorsal raphe; f, fornix; fr, fasciculus retroflexus; GP, globus pallidus; ic, internal capsule; IP, interpeduncular nucleus; $\mathrm{LDTg}$, laterodorsal tegmental nucleus; $\mathrm{LH}$, lateral hypothalamic area; LHb, lateral habenula; $\mathrm{MHb}$, medial habenula; $M M$, mammillary body; $M R$, median raphe; $0 \mathrm{t}$, optictract; $\mathrm{Pa}$, paraventricular hypothalamicnucleus; $\mathrm{PAG}$, periaqueductal gray; $\mathrm{PB}$, parabrachial nucleus; $\mathrm{Pf}$, parafascicularnucleus; $\mathrm{PH}$, posterior hypothalamic nucleus; $\mathrm{PnC}$, caudal field of pontine reticularformation; $\mathrm{PnO}$, oral field of pontine reticular formation; Pr, prehypoglossal nucleus; PPTg, pedunculopontine tegmental nucleus; R, red nucleus; RIP, raphe interpositus; scp, superior cerbellar peduncle; $\mathrm{SNr}$, substantia nigra reticulata; STN, subthalamic nucleus; SUM, suparmammillary nucleus; TC, tuber cinereum; VEn, ventral endopiriform nucleus; VMH, ventromedial hypothalamic nucleus; $x s c p$, decussatio of scp; ZI, zona incerta; III, third ventricle; 4 v, fourth ventricle; $7 \mathrm{n}$, facial nerve. Scale bar: (in D) DI, $300 \mu \mathrm{m} ; \mathbf{K I}, 200 \mu \mathrm{m}$; DII, DIII, KII, KIII, $20 \mu \mathrm{m}$.
} 
rograde labeling in the caudate-putamen, globus pallidus, subthalamic nucleus, and ventral tegmental nucleus. Although none of the injection sites of the cases used to analyze the distribution of VGLUT2 and VGLUT3 VTA-projecting neurons involved the interpeduncular nucleus (Table 1, Fig. 2), some occult spread of tracer into that structure might have occurred, resulting in some false-positive VGLUT2 and VGLUT3 VTA-projecting neurons in the dorsal and median raphe. Involvement of the fasciculus retroflexus in some of our injections may have resulted in falsepositive labeling of VGLUT1- and VGLUT2-VTA-projecting neurons in the medial and lateral habenula, respectively. However, the same percentage of VGLUT2-VTA-projecting neurons was detected in the lateral habenula whether the injection sites involved the fasciculus retroflexus (cases 05179 and 06151, 6.8 and $2.3 \%$, respectively) or not (cases 06056 and $06084,6.4$ and $3.8 \%$, respectively). Although it remains unclear at present whether fibers from the medial habenula terminate in the VTA or just pass through (for discussion, see Geisler and Zahm, 2005), VGLUT1-VTAprojecting neurons in the medial habenula were plotted and illustrated (Fig. 3).

Probes were generated against the $\mathrm{C}$ termini of the protein-coding and adjacent $3^{\prime}$ untranslated regions of the three VGLUTs, where the nucleotide sequences differ. Each probe exhibited a labeling pattern distinct from the other two and in good accord with previous isotopic (Hisano et al., 2000; Fremeau et al., 2001; Herzog et al., 2001, 2004) and nonisotopic (Hur and Zaborszky, 2005) in situ hybridization studies. Hybridization conditions first were adjusted to eliminate background or weak "Nissling" of structures known to lack VGLUT mRNA (Hisano et al., 2000; Fremeau et al., 2001; Herzog et al., 2001, 2004; Hur and Zaborszky, 2005). Increasing the concentration of VGLUT3 cRNA produced weak labeling in structures positive for VGLUT2 mRNA, such as the laterodorsal tegmental nucleus, various thalamic nuclei, and the superior colliculus. Similarly, increasing the concentrations of VGLUT1 and VGLUT2 cRNAs produced weak labeling, respectively, in the hypothalamus and all cortical layers. Because these weak signals were observed only with high concentrations in structures positive for other VGLUT mRNAs, we cautiously interpreted them as cross-reactivity, possibly reflecting homologous sequences in the C-terminal regions of the three probes. True labeling of low levels of mRNA cannot be ruled out, however.

Although before this study VGLUT2 mRNA labeling in the median raphe had not been described, we are confident of the validity of this observation. First, the VGLUT2 mRNA labeling in the median raphe was among the strongest in the brain. Second,
Table 4. Distribution of VGLUT3 mRNA-positive, VTA-projecting neurons

\begin{tabular}{lll}
\hline Structure & $\begin{array}{l}\text { Percentage } \\
\text { of total }\end{array}$ & $\begin{array}{l}\text { Percentage of retrogradely labeled neurons } \\
\text { positive for VGLUT3 mRNA }\end{array}$ \\
\hline $\begin{array}{l}\text { Dorsal raphe } \\
\text { Median raphe }\end{array}$ & $72.5 \pm 5.9$ & $64.0 \pm 5.3$ \\
Others $^{a}$ & $19.0 \pm 5.2$ & $21.8 \pm 4.2$ \\
\hline
\end{tabular}

Values are percentages of total VGLUT3 mRNA-positive, VTA-projecting neurons and are listed in descending order; $n=5$ rats $(86,87,119,145$, and 163 double-labeled neurons per series of sections, respectively, were plotted). n.d. Not determined.

Includes the anterior nucleus of the medial bed nucleus of the stria terminalis, lateral habenula, and pontine central gray. 

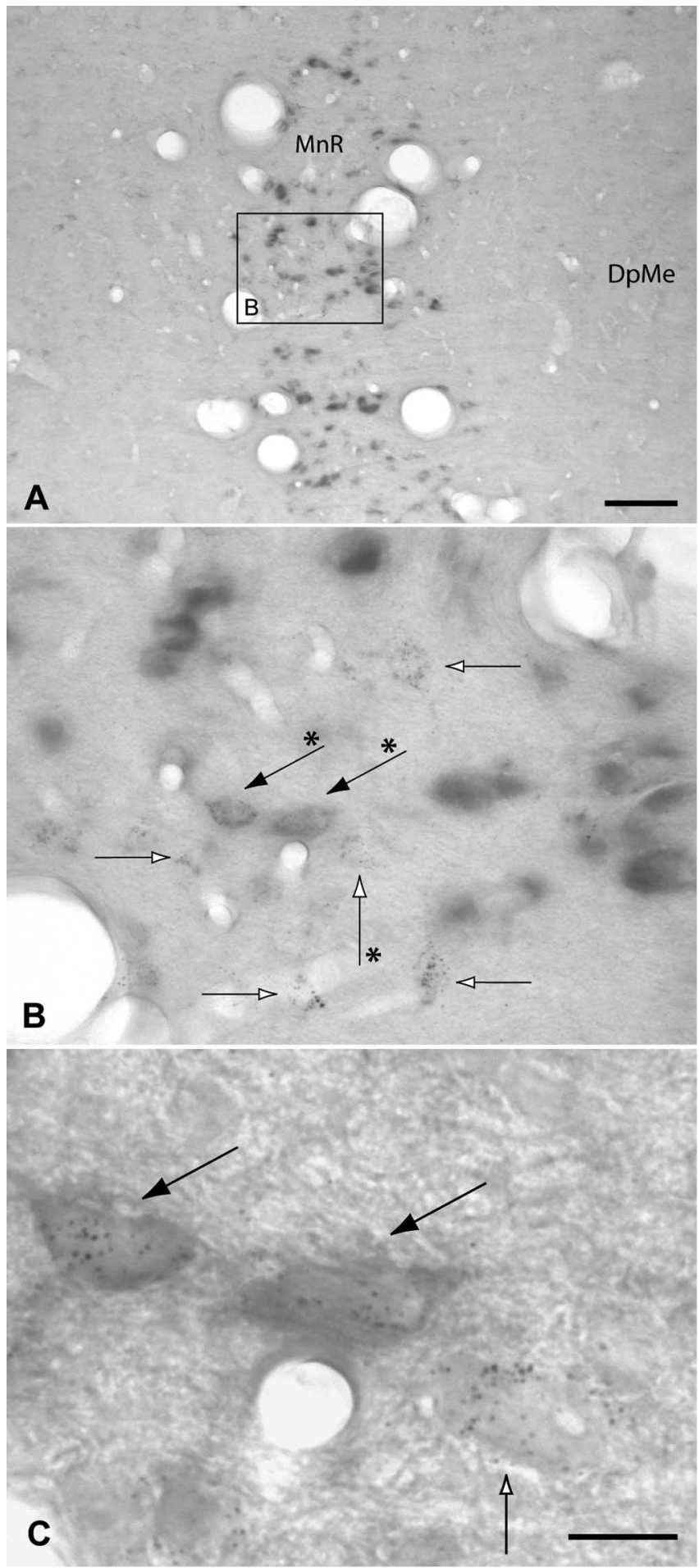

Figure 9. A-C, VGLUT3 mRNA was strongly expressed in neurons of the median raphe $(\mathrm{MnR})$, whereas no signal was detected in the adjacent deep field of the mesencephalic reticular formation (DpME). Although many retrogradely labeled neurons were present in the median raphe (open arrows), only a few of them expressed both markers (black arrows). C is an enlargement of $\boldsymbol{B}$, illustrating the double-labeled and retrogradely labeled neurons that are marked in $B$ with arrows indicated by asterisks. Scale bars: $A, 100 \mu \mathrm{m} ; \boldsymbol{B}, 20 \mu \mathrm{m} ; \boldsymbol{C}, 10 \mu \mathrm{m}$.

VGLUT2 mRNA labeling was absent in dorsal raphe and striatal cholinergic neurons, which express high levels of VGLUT3 mRNA, militating against detectable cross-reactivity in the hybridization conditions we used.
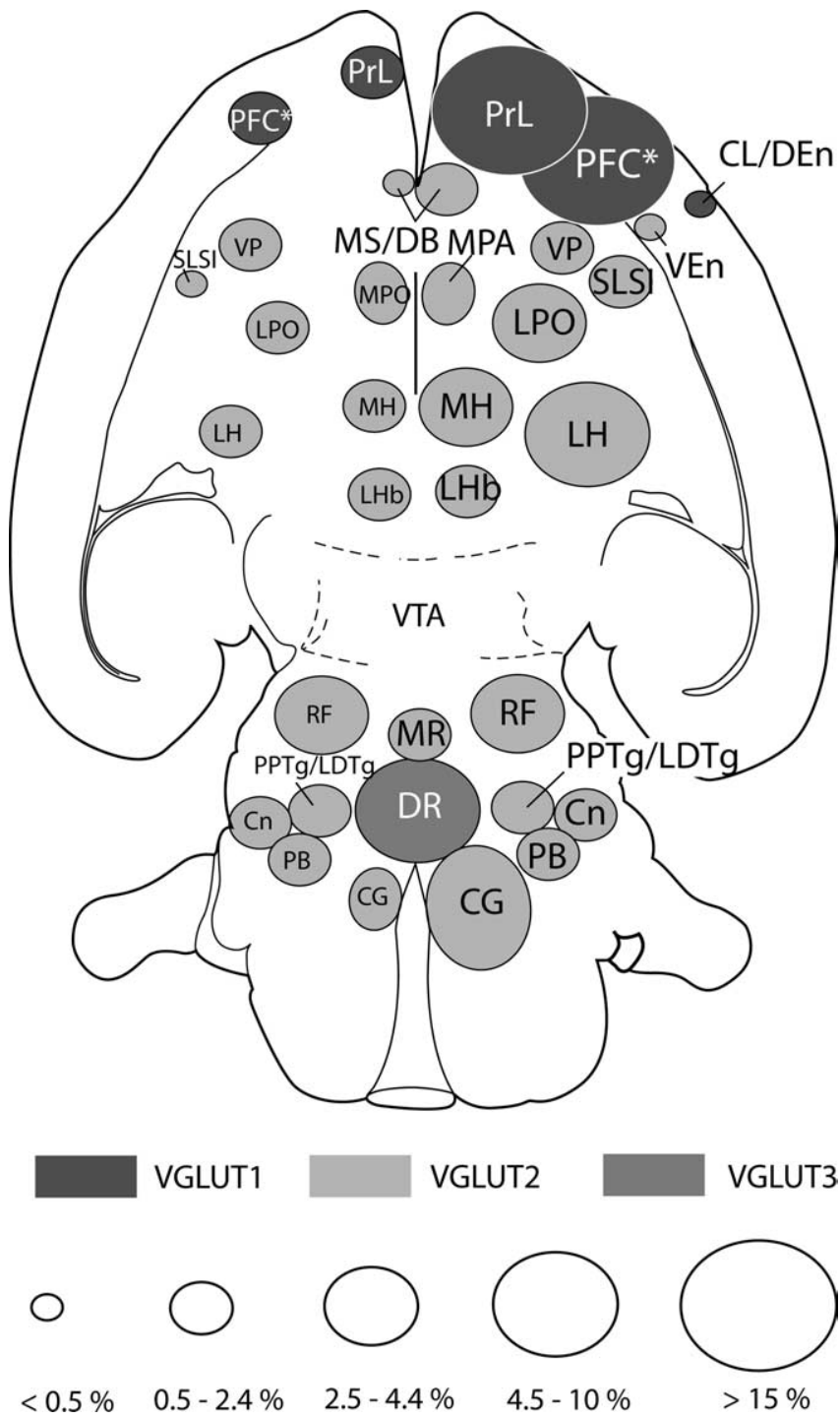

Figure 10. Summary diagram depicting glutamatergic projections to the VTA. The brain areas containing VGLUT1 (dark gray), VGLUT2 (light gray), or VGLUT3 (slate) mRNA-positive, VTA-projecting neurons are indicated separately. The sizes of ovals reflect the hypothetical proportion of the VGLUT-VTA-projecting neurons in those structures relative to all VGLUT mRNA-positive, VTA-projecting neurons. To arrive at these figures, the averages of VGLUT1, VGLUT2, and VGLUT3 mRNA-positive, VTA-projecting neurons of all cases ( $n=12$ rats) were added and taken as the total number of neurons, and the proportions of the VGLUT in every structure were calculated from it. The right side of the figure is ipsilateral to the injections. $\mathrm{Cl} /$ Den, Claustrum/dorsal endopiriform area; $\mathrm{CG}$, central gray; $\mathrm{Cn}$, cuneiform nucleus; $\mathrm{DR}$, dorsal raphe; $L H$, lateral hypothalamic area; $L H b$, lateral habenula; $L P O$, lateral preoptic area; $M H$, medial hypothalamus; MPA, medial preoptic area; MR, median raphe; MS/DB, medial septum/ diagonal band complex; $\mathrm{PB}$, parabrachial nucleus; $\mathrm{PFC}^{*}$, prefrontal cortex, prelimbic cortex not included; PPTg/LDTg, pedunculopontine and laterodorsal tegmental nuclei; PrL, prelimbic cortex; RF, reticular formation; SLSI, sublenticular substantia innominata; VEn, ventral endopiriform area; $V P$, ventral pallidum.

\section{Functional considerations}

The present study reveals that glutamatergic inputs converge in the VTA from almost all of the many brain structures projecting there, supporting the previously articulated hypothesis that the VTA functions by integrating various and diverse bits of information (Oades and Halliday, 1987; Geisler and Zahm 2005, $2006 a, b)$. In view of this anatomical organization, it seems reasonable to conceive that the activity of the VTA reflects the ongoing activities of various combinations of afferents, which are 
triggered in relation to changing patterns of sensorimotor stimuli and inflections of body state.

Insofar as VTA neurons have long, sparsely branching dendrites (Phillipson, 1979) contacted by many afferent fibers, which, in general, arborize relatively sparsely within the VTA (Geisler and Zahm, 2005), the most robust firing of VTA neurons should be elicited by simultaneous activation of diverse glutamatergic afferents. This idea is consistent with the observation of a reduced activation of VTA dopamine neurons after the omission of one of two reward-predicting stimuli previously delivered simultaneously (Schultz, 1986). The demonstration that simultaneous activation of two inputs to the VTA produces an additive increase in dopamine cell firing (Lodge and Grace, 2006b) also is consistent with this conceptual framework. It also should be noted that, whereas the projections from the prefrontal cortex are essentially entirely glutamatergic (Fig. 3, Table 2), those from subcortical structures comprise a mixture of different phenotypes. Thus, subcortical VTA-projecting neurons expressing VGLUT2 and VGLUT3 mRNA intermingle with (most likely GABAergic and/or peptidergic) VTA-projecting neurons lacking VGLUTs (Figs. 4-9; Tables 3, 4), consistent with a wide range of potential subcortical influences on VTA activity.

To summarize, the present study has revealed that the VTA receives glutamatergic afferents from many sources, consistent with the hypothesis that the VTA functions by integrating multifarious bits of information. It will be important to investigate which glutamatergic afferents target which neuron populations in the VTA, as has recently been done so elegantly for afferents from the prefrontal cortex and laterodorsal tegmental nucleus (Carr and Sesack, 2000; Omelchenko and Sesack, 2005).

\section{References}

Bellocchio EE, Reimer RJ, Fremeau Jr RT, Edwards RH (2000) Uptake of glutamate into synaptic vesicles by an inorganic phosphate transporter. Science 289:957-960.

Carr DB, Sesack SR (2000) Projections from the rat prefrontal cortex to the ventral tegmental area: target specificity in the synaptic associations with mesoaccumbens and mesocortical neurons. J Neurosci 20:3864-3873.

Charara A, Smith Y, Parent A (1996) Glutamatergic inputs from the pedunculopontine nucleus to midbrain dopaminergic neurons in primates: Phaseolus vulgaris-Leucoagglutinin anterograde labeling combined with postembedding glutamate and GABA immunohistochemistry. J Comp Neurol 364:254-266.

Charlety PJ, Grenhoff J, Chergui K, De La Chapelle B, Buda M, Svensson TH, Chouvet G (1991) Burst firing of mesencephalic dopamine neurons is inhibited by somatodendritic application of kynurenate. Acta Physiol Scand 142:105-112.

Chergui K, Charlety PJ, Akaoka H, Saunier CF, Brunet J-L, Buda M, Svensson TH, Chouvet G (1993) Tonic activation of NMDA receptors causes spontaneous burst discharge of rat midbrain dopamine neurons in vivo. Eur J Neurosci 5:137-144.

Coizet F, Comoli E, Westby GW, Redgrave P (2003) Phasic activation of substantia nigra and the ventral tegmental area by chemical stimulation of the superior colliculus: an electrophysiological investigation in the rat. Eur J Neurosci 17:28-40.

Floresco SB, West AR, Ash B, Moore H, Grace AA (2003) Afferent modulation of dopamine neurons firing differentially regulates tonic and phasic dopamine transmission. Nat Neurosci 6:968-973.

Fremeau Jr RT, Troyer MD, Pahner I, Nygaard GO, Tran CH, Reimer RJ, Bellocchio EE, Fortin D, Storm-Mathisen J, Edwards RH (2001) The expression of vesicular glutamate transporters defines two classes of excitatory synapse. Neuron 31:247-260.

Fremeau Jr RT, Burman J, Qureshi T, Tran CH, Proctor J, Johnson J, Zhang H, Sulzer D, Copenhagen DR, Storm-Mathisen J, Reimer RJ, Chaudhry FA, Edwards RH (2002) The identification of vesicular glutamate transporter 3 suggests novel modes of signaling by glutamate. Proc Natl Acad Sci USA 99:14488-14493.
Fremeau Jr RT, Voglmaier S, Seal RP, Edwards RH (2004) VGLUTs define subsets of excitatory neurons and suggest novel roles for glutamate. Trends Neurosci 27:98-103.

Geisler S, Zahm DS (2005) Afferents of the ventral tegmental area in the rat-anatomical substratum for integrative functions. J Comp Neurol 490:270-294.

Geisler S, Zahm DS (2006a) On the retention of neurotensin in the ventral tegmental area (VTA) despite destruction of the main neurotensinergic afferents of the VTA-implications for the organization of forebrain projections to the VTA. Brain Res 1087:87-104.

Geisler S, Zahm DS (2006b) Neurotensin afferents of the ventral tegmental area in the rat: [1] re-examination of their origins and [2] responses to acute psychostimulant and antipsychotic drug administration. Eur J Neurosci 24:116-134.

Georges F, Aston-Jones G (2001) Potent regulation of midbrain dopamine neurons by the bed nucleus of the stria terminalis. J Neurosci 21:RC160(1-6).

Georges F, Aston-Jones G (2002) Activation of ventral tegmental area cells by the bed nucleus of the stria terminalis: a novel excitatory amino acid input to midbrain dopamine neurons. J Neurosci 22:5173-5187.

Grace AA, Bunney BS (1984) The control of firing pattern in nigral dopamine neurons: burst firing. J Neurosci 4:2877-2890.

Gras C, Herzog E, Bellenchi GC, Bernard V, Ravassard P, Pohl M, Gasnier B, Giros B, El Mestikawy S (2002) A third vesicular glutamate transporter expressed by cholinergic and serotoninergic neurons. J Neurosci 22:5442-5451.

Harris GC, Aston-Jones G (2003) Critical role for ventral tegmental glutamate in preference for a cocaine-conditioned environment. Neuropsychopharmacology 28:73-76.

Harris GC, Wimmer M, Byrne R, Aston-Jones G (2004) Glutamateassociated plasticity in the ventral tegmental area is necessary for conditioning environmental stimuli with morphine. Neuroscience 129:842-847.

Herzog E, Bellenchi GC, Gras C, Bernard V, Ravassard P, Bedet C, Gasnier B, Giros B, El Mestikawy S (2001) The existence of a second vesicular glutamate transporter specifies subpopulations of glutamatergic neurons. J Neurosci 21:RC181(1-6).

Herzog E, Gilchrist J, Gras C, Muzerelle A, Ravassard P, Giros B, Gaspar P, El Mestikawy S (2004) Localization of VGLUT3, the vesicular glutamate transporter type 3 , in the rat brain. Neuroscience 123:983-1002.

Hisano S, Hoshi K, Ikeda Y, Maruyama D, Kanemoto M, Ichijo H, Kojima I, Takeda J, Nogami H (2000) Regional expression of a gene encoding a neuron-specific $\mathrm{Na}+$-dependent inorganic phosphate cotransporter (DNPI) in the rat forebrain. Mol Brain Res 83:34-43.

Hur EE, Zaborszky L (2005) Vglut2 afferents to the medial prefrontal and primary somatosensory cortices: a combined retrograde tracing in situ hybridization. J Comp Neurol 483:351-373.

Hyland BI, Reynolds JNJ, Hay J, Perk CG, Miller R (2002) Firing modes of midbrain dopamine cells in the freely moving rat. Neuroscience 114:475-495.

Johnson SW, Seutin V, North RA (1992) Burst firing in dopamine neurons induced by $\mathrm{N}$-methyl-D-aspartate: role of electrogenic sodium pump. Science 258:665-667.

Jongen-Relo AL, Amaral DG (2000) Double labeling technique using WGA-apoHRP-gold as a retrograde tracer and non-isotopic in situ hybridization histochemistry for the detection of mRNA. J Neurosci Methods 101:9-17.

Kalivas PW, Churchill L, Klitenick MA (1993) GABA and enkephalin projection from the nucleus accumbens and ventral pallidum to the ventral tegmental area. Neuroscience 57:1047-1060.

Lodge DJ, Grace AA (2006a) The laterodorsal tegmentum is essential for burst firing of ventral tegmental area dopamine neurons. Proc Natl Acad Sci USA 103:5167-5172.

Lodge DJ, Grace AA (2006b) The hippocampus modulates dopamine neuron responsivity by regulating the intensity of phasic neuron activation. Neuropsychopharmacology 31:1356-1361.

Murase S, Grenhoff J, Chouvet G, Gonon FG, Svensson TH (1993) Prefrontal cortex regulates burst firing and transmitter release in rat mesolimbic dopamine neurons studied in vivo. Neurosci Lett 157:53-56.

Oades RD, Halliday GM (1987) Ventral tegmental (A10) system: neurobiology. 1. Anatomy and connectivity. Brain Res Rev 12:117-165. 
Omelchenko N, Sesack SR (2005) Laterodorsal tegmental projections to identified cell populations in the rat ventral tegmental area. J Comp Neurol 483:217-235.

Paxinos G, Watson C (1998) The rat brain in stereotaxic coordinates, Ed 4. San Diego: Academic.

Phillipson OT (1979) A Golgi study of the ventral tegmental area of Tsai and interfascicular nucleus in the rat. J Comp Neurol 187:99-116.

Schafer MK, Varoqui H, Defamie N, Weihe E, Erickson JD (2002) Molecular cloning and functional identification of mouse vesicular glutamate transporter 3 and its expression in subsets of novel excitatory neurons. J Biol Chem 277:50734-50748.

Schultz W (1986) Responses of midbrain dopamine neurons to behavioral trigger stimuli in the monkey. J Neurophysiol 56:1439-1461.

Schultz W (1998) Predictive reward signal of dopamine neurons. J Neurophysiol 80:1-27.

Sesack SR, Pickel VM (1992) Prefrontal cortical efferents in the rat synapse on unlabeled neuronal targets of catecholamine terminals in the nucleus accumbens septi and on dopamine neurons in the ventral tegmental area. J Comp Neurol 320:145-160.

Sun WL, Rebec GV (2005) Ionotropic glutamate receptors in the ventral tegmental area regulate cocaine-seeking behavior in rats. Neuropsychopharmacology 30:2073-2081.

Takamori S (2006) VGLUTs: "exciting” times for glutamate research? Neurosci Res 55:343-351.

Takamori S, Rhee JS, Rosenmund C, Jahn R (2000) Identification of a vesicular glutamate transporter that defines a glutamatergic phenotype in neurons. Nature 407:189-1894.

Takamori S, Rhee JS, Rosenmund C, Jahn R (2001) Identification of differentiation-associated brain-specific phosphate transporter as a second vesicular glutamate glutamate transporter (VGLUT2). J Neurosci 21:RC182(1-6).

Varoqui H, Schafer MK, Zhu H, Weihe E, Erickson JD (2002) Identification of the differentiation-associated $\mathrm{Na}+$ /PI transporter as a novel vesicular glutamate transporter expressed in a distinct set of glutamatergic synapses. J Neurosci 22:142-155.

Vorel SR, Liu X, Hayes RJ, Spector JA, Gardner EL (2001) Relapse to cocaine-seeking after hippocampal theta burst stimulation. Science 292:1175-1178.

Wise RA (2004) Dopamine, learning and motivation. Nat Rev 5:1-12. 